\title{
苯并噻唑基荧光探针的设计、合成与应用研究进展
}

\author{
蒋 凯 ${ }^{a}$ 曹 梁 ${ }^{a}$ 郝志峰 $*, b$ 陈美燕 ${ }^{a}$ 程洁銮 $a$ \\ 李 晓 ${ }^{a}$ 肖萍 $a$ 陈亮 ${ }^{a}$ 汪朝阳 $*$, $a$ \\ ( ${ }^{a}$ 华南师范大学化学与环境学院 教育部环境理论化学重点实验室 广州 510006) \\ ( ${ }^{b}$ 广东工业大学轻工化工学院 广州 510006)
}

\begin{abstract}
摘要 苯并噻唑基团具有特征苂光，且结构中含有 $\mathrm{N}$ 和 $\mathrm{S}$ 杂原子，因而在荧光探针结构中常被视为荧光基团与识别基团， 承担荧光信号输出与提供结合位点的重要作用. 利用苯并噻唑基团的特点来设计合成性能更佳的荧光探针已逐渐成为 苂光探针研究领域中瞩目的焦点. 对近几年来报道的苯并噻唑基荧光探针按不同的检测对象分为金属阳离子、阴离子、 含硫化合物及其它物种等四大类, 综述了其设计、合成、作用机理及其相关检测应用, 并展望了其未来的发展趋势. 关键词 苯并噻唑; 苂光探针; 设计与合成; 检测应用; 进展
\end{abstract}

\section{Research Progress in Design, Synthesis and Application of Benzothiazole-Based Fluorescent Probes}

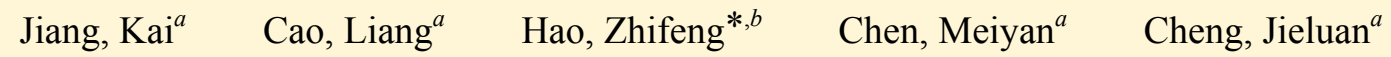 \\ $\mathrm{Li}, \mathrm{Xiao}^{a} \quad \mathrm{Xiao}^{a}$ Ping $^{a} \quad$ Chen, Liang $^{a} \quad$ Wang, Zhaoyang ${ }^{*, a}$ \\ ( ${ }^{a}$ School of Chemistry and Environment, Key Laboratory of Theoretical Chemistry of Environment, Ministry of Education, \\ South China Normal University, Guangzhou 510006) \\ ( ${ }^{b}$ School of Chemical Engineering and Light Industry, Guangdong University of Technology, Guangzhou 510006)
}

\begin{abstract}
Benzothiazole group is of characteristic fluorescence, and each also contains two heteroatoms (N and S). Hence, benzothiazole group is always viewed as fluorophore and recognition moiety in structure of fluorescent probes, playing an essential role on provision of fluorescence signal and binding sites. Recently, utilizing these properties of benzothiazole moiety to design and synthesize better fluorescent probes has gradually become one of focuses in fluorescent detection research. Classifying benzothiazole-based fluorescent probes into four main categories according to different analytes, such as metal cations, anions, sulfur-containing compounds and other species, the design, synthesis, detecting mechanism and relevant application of these probes are reviewed. And their development tendency in the future is prospected.
\end{abstract}

Keywords benzothiazole; fluorescent probe; design and synthesis; detecting application; progress

苯并噻唑类化合物长期以来备受科学家们的关注, 尤其在生物制药 ${ }^{[1,2]}$ 和医疗诊断 ${ }^{[3]}$ 领域有重要应用. 其 实, 就苯并噻唑基团而言, 由于刚性的平面结构与离域 的大 $\pi$ 键的存在, 使苯并噻唑类化合物荧光量子产率较 高, 近年来在光电材料 ${ }^{[4]}$ 和荧光探针 ${ }^{[5 \sim 7]}$ 制备方面受到 重视, 特别是其结构中含有的 $\mathrm{N} 、 \mathrm{~S}$ 原子可与金属配位
等作用 ${ }^{[8,9]}$, 使它们在苂光探针方面的应用得到了迅猛 的发展 ${ }^{[9,10]}$. 鉴于此, 本文对近期苯并噻唑基苂光探针 的设计、合成与应用情况方面的进展进行了综述.

\section{1 应用于金属阳离子的检测}

金属离子含量超标对生态环境造成严重的污染，给

\footnotetext{
* Corresponding authors. E-mail: wangzy@scnu.edu.cn; haozhifeng3377@163.com Received March 24, 2017; revised May 14, 2017; published online May 24, 2017. Project supported by the Guangzhou Science and Technology Project Scientific Special (No. 201607010251), the Applied Science and Technology Research and Development Special Foundation of Guangdong Province (No. 2016B090930004 ), the Undergraduates Innovation Project of South China Normal University (No. 20161415), the Natural Science Foundation of Guangdong Province (No. 2014A030313429) and the Guangdong Provincial Science and Technology Project (No. 2017A010103016).

广州市科技计划科学研究专项(No. 201607010251)、广东省应用型科技研发专项资金(No. 2016B090930004)、华南师范大学大创计划(No. 20161415)、 广东省自然科学基金(No. 2014A030313429)及广东省科技计划(No. 2017A010103016)资助项目.
} 
人体健康带来巨大的危害. 因此, 设计、合成可应用于 金属离子检测的苂光探针受到人们的重视. 目前, 有很 多文献报道了基于苯并噻唑化合物的荧光探针分子用 于检测 $\mathrm{Hg}^{2+}, \mathrm{Cu}^{2+}, \mathrm{Fe}^{3+}, \mathrm{Zn}^{2+}$ 等金属离子 ${ }^{[11]}$.

\section{1 检测录离子}

利用苯并噻唑基苂光探针检测 $\mathrm{Hg}^{2+}$, 是近年来荧 光探针研究领域的热点之一 ${ }^{[12,13]}$. 例如, Ju 等 ${ }^{[14]}$ 以 2-氨 基苯硫酚(1)为原料，设计、合成了探针 2 (Eq. 1), 当其 中具有特殊配位作用的大环选择性地固定 $\mathrm{Hg}^{2+}$ 时, 引 起原本由苯并噻唑基团提供的 $416 \mathrm{~nm}$ 处特征发射峰对 应的淡黄色荧光猝灭, 故能实现对 $\mathrm{Hg}^{2+}$ 的专一识别.

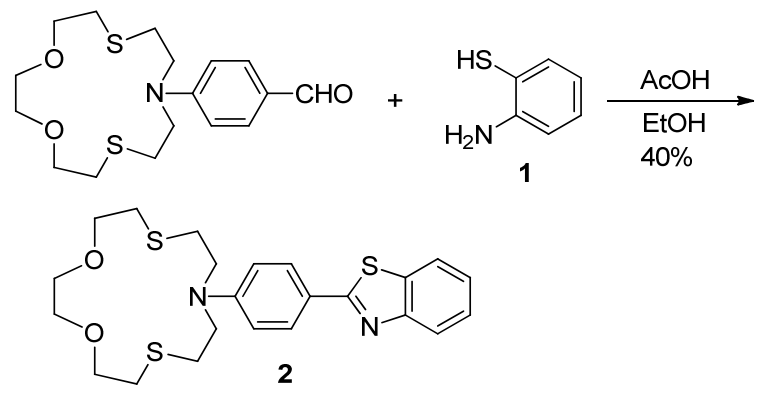

Sahana 等 ${ }^{[15]}$ 也将苯并噻唑基团作为荧光团引入探 针分子, 以 1 和水杨醛 3 等为原料, 经多步反应合成了 “turn on” 型探针分子 5 (Scheme 1). 其中, NH、酚羟基 和喹啉基团上 $\mathrm{N}$ 原子与 $\mathrm{Hg}^{2+}$ 配位, 在较宽 $\mathrm{pH}$ 范围的缓 冲溶液体系内形成了具有黄色荧光的金属配合物, 而且 可在 Hela 细胞内实现对 $\mathrm{Hg}^{2+}$ 的检测.

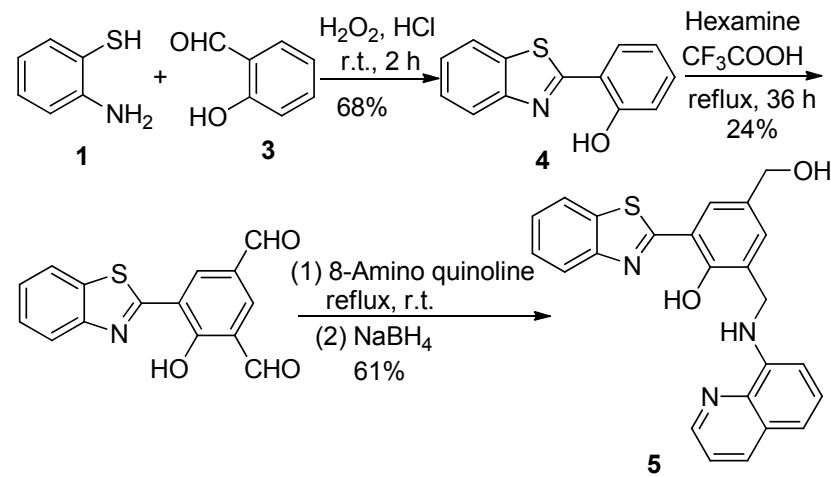

图式 1 探针 5 的合成

Scheme 1 Synthesis of probe 5

不仅能够利用苯并噻唑基团充当特征苂光团的功 能设计探针分子, 还可利用其提供配位原子的特点设 计、合成探针 ${ }^{[16 ~ 18]}$. 例如, 梅群波等 ${ }^{[19]}$ 合成了一系列基 于 2-噻吩基苯并噻唑的探针分子(如化合物 6), 它们都 能与 $\mathrm{Hg}^{2+}$ 发生配位作用. 当探针 6 以 $1: 1$ 配位时(Eq. 2 ), 在可见光下观察到溶液由无色变为黄色, 故可实现 可见光下的裸眼识别. 不仅如此, 其苂光光谱也呈现着
比率型的变化，最大发射波长从 $468 \mathrm{~nm}$ 红移至 $567 \mathrm{~nm}$, 苂光颜色由蓝变黄, 检测限低达 $3.98 \times 10^{-6} \mathrm{~mol} \cdot \mathrm{L}^{-1}$.

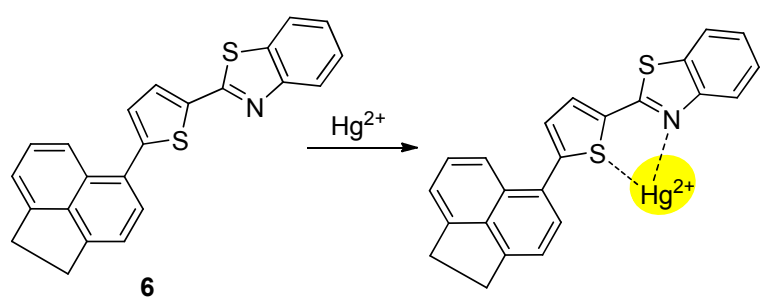

把富电子基团与苯并噻唑基团相连，也是构建苂光 探针的一种手段. 例如, Razi 等 ${ }^{[20]}$ 以化合物 1 为原料，首 先合成了中间体 7, 再进一步通过多步反应, 得到 $D-\pi-A$ 型探针分子 8 (Scheme 2). 由于分子内存在着由 咪唑基团到苯并噻唑基团的分子内电荷转移(ICT)现象, 当其与 $\mathrm{Hg}^{2+}$ 以 $1: 1$ 形成配合物后, 配位作用使得 ICT 机制受阻，探针 8 上的电荷分布发生变化，苂光发射峰 蓝移 $28 \mathrm{~nm}$, 菼光量子产率从 0.24 提高至 0.61 . 因此，这 种仅对 $\mathrm{Hg}^{2+}$ 的螯合增强苂光 $(\mathrm{CHEF})$ 效应使探针 $\mathbf{8}$ 可以 选择性地检测 $\mathrm{Hg}^{2+}$.
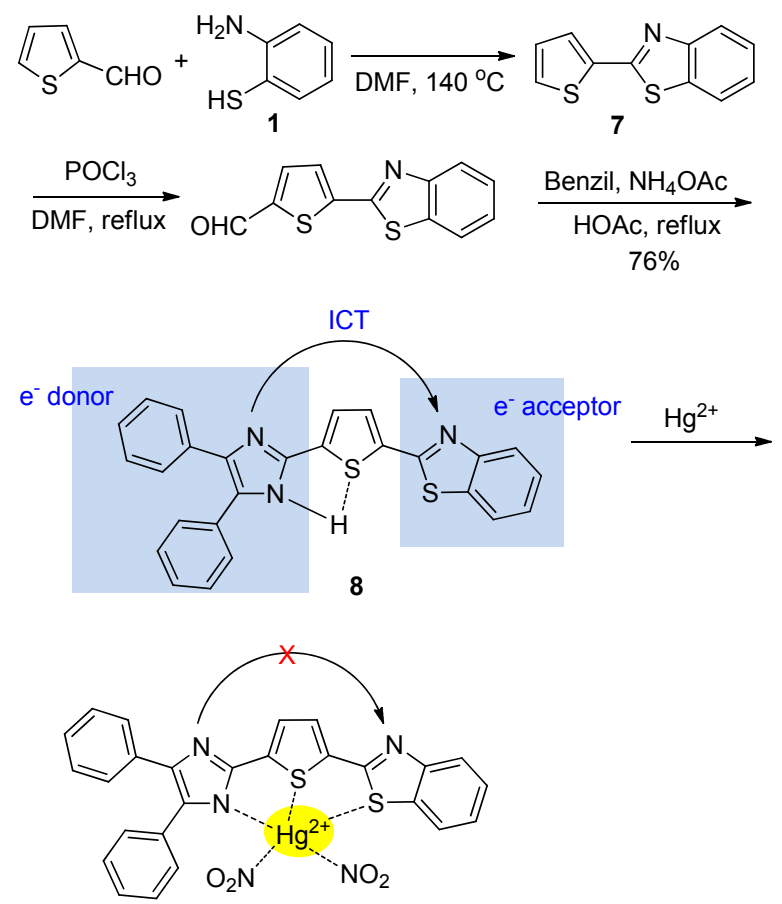

图式 2 探针 $\mathbf{8}$ 的合成与作用机理

Scheme 2 Synthesis of probe 8 and its interaction mechanism

有别于上述探针分子基于与 $\mathrm{Hg}^{2+}$ 发生配位作用形 成配合物从而改变荧光性质的原理, 反应型 $\mathrm{Hg}^{2+}$ 探针 则是通过与 $\mathrm{Hg}^{2+}$ 的反应，改变自身结构，使原有荧光性 质发生变化来达到检测的目的 ${ }^{[21]}$. 其中, 多数反应型探 针都是基于常见的激发态分子内质子转移(ESIPT)机制 设计的 ${ }^{[22]}$. 
例如，Ahn 等 ${ }^{[23]}$ 把乙烯醚基团引入到苯并噻唑基苯 上, 可得到探针 9 , 其与二价录盐发生羟录化反应而水 解, 生成类似典型的 ESIPT型苂光探针结构 2-(2-羟基苯 基)-苯并噻唑(HBT)的化合物 10. 在 $365 \mathrm{~nm}$ 激发波长的 激发下时, 10 的苯酚结构互变为具有更长发射波长和更 强苂光的醌式结构(Scheme 3). 由于是经羟录化而水解 的特殊反应机理，该探针具有良好的选择性.

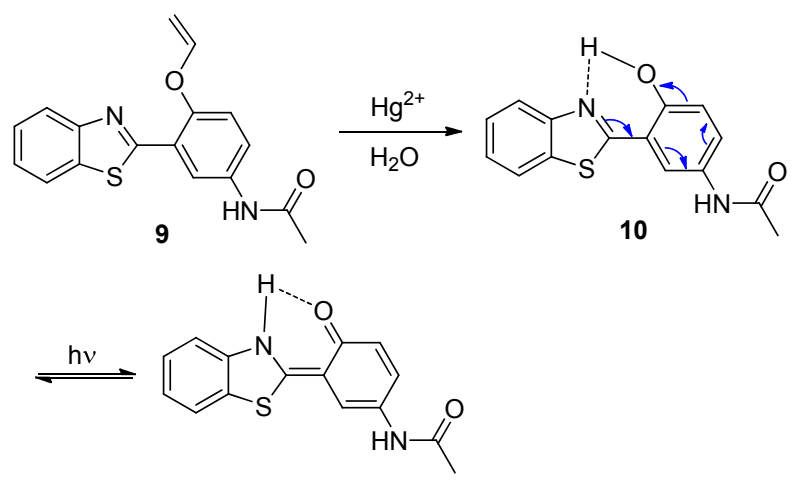

图式 3 探针 9 的作用机理

Scheme 3 Interaction mechanism of probe 9

在国内, 刘伟生课题组 ${ }^{[24]}$ 则将烯丙基接入到 2-苯并 噻唑基苯酚(4)成醚, 经 Claisen 重排后得到了反应型探 针分子 11 (Scheme 4). 往含有 $0.5 \% \mathrm{CH}_{3} \mathrm{CN}$ 的 11 的磷酸 盐缓冲溶液(PBS)加入 $\mathrm{HgCl}_{2}$, 化合物 $\mathbf{1 2}$ 的生成阻止了 ESIPT 过程的发生, 导致荧光光谱发生变化, 溶液荧光 颜色由绿变蓝. 但是, 随着硼氢化钠的加入, 化合物 $\mathbf{1 2}$ 可回到原来探针 11 的结构, 使荧光颜色复原, 故能实现 可逆检测.
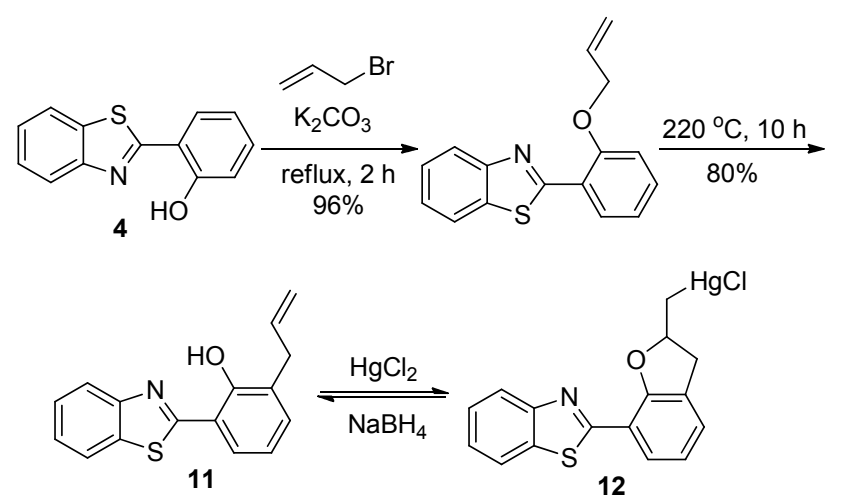

图式 4 探针 11 的合成与作用机理

Scheme 4 Synthesis of probe $\mathbf{1 1}$ and its interaction mechanism

除此之外, Chang 等 ${ }^{[25]}$ 根据仅录离子可催化硫代酰 胺水解为酰胺的反应特点, 设计合成了脱 $\mathrm{HgS}$ 的另一类 反应型 $\mathrm{Hg}^{2+}$ 探针分子 $\mathbf{1 3}$ (Scheme 5), 其对 $\mathrm{Ni}^{2+}, \mathrm{Cu}^{2+}$, $\mathrm{Cd}^{2+}$ 等其它过渡金属均无响应，显示出对 $\mathrm{Hg}^{2+}$ 具有良 好的选择性.
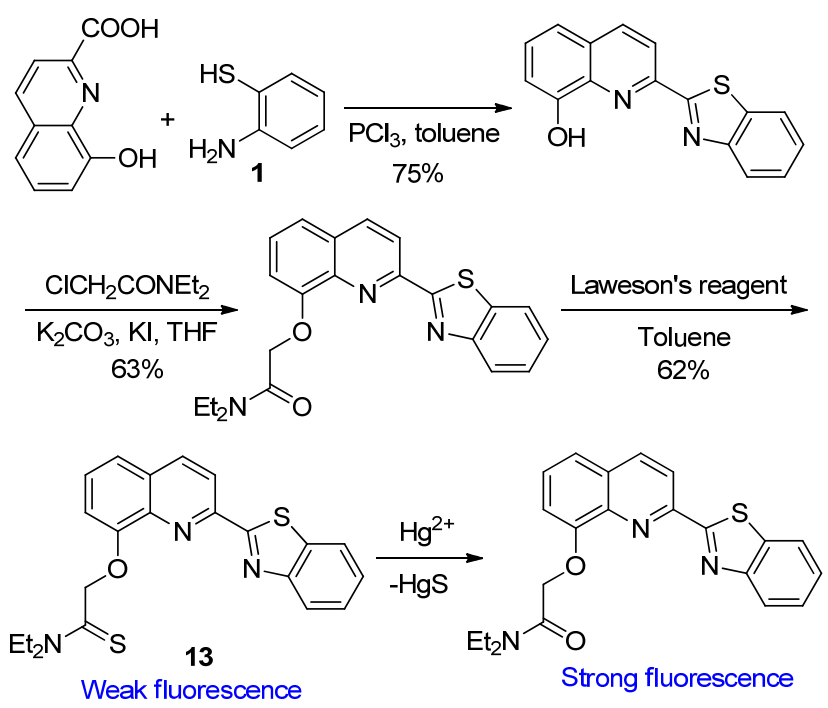

图式 5 探针 13 的合成与作用机理

Scheme 5 Synthesis of probe $\mathbf{1 3}$ and its interaction mechanism

\section{2 检测铜离子}

$\mathrm{Cu}^{2+}$ 的检测一直受到关注 ${ }^{[26]}$, 用于检测 $\mathrm{Cu}^{2+}$ 的苯 并噻唑基探针也常被报道 ${ }^{[27,28]}$. 其中，较为常见的是涉 及 HBT 结构的 ESIPT 型苂光探针 ${ }^{[21]}$. 例如, Tang 等 ${ }^{[29]}$ 合成了的反应型探针 14 , 利用 $\mathrm{Cu}^{2+}$ 对化合物 14 的催化 水解作用，可生成 ESIPT 型结构的分子 15 (Scheme 6), 其对 $\mathrm{Cu}^{2+}$ 检测限达 $1.34 \times 10^{-6} \mathrm{~mol} \cdot \mathrm{L}^{-1}$ ，低于美国国家 环境保护局饮用水中 $2 \times 10^{-5} \mathrm{~mol} \cdot \mathrm{L}^{-1}$ 的 $\mathrm{Cu}^{2+}$ 浓度限度 要求.

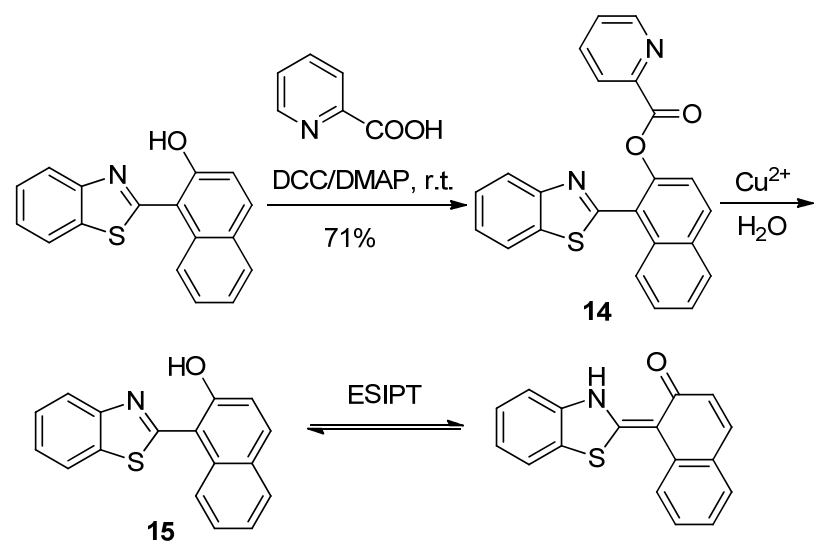

图式 6 探针 14 的合成与作用机理

Scheme 6 Synthesis of probe $\mathbf{1 4}$ and its interaction mechanism

罗丹明类化合物常被设计成荧光探针，当其结构改 造中引入 $\mathrm{HBT}$ 结构时, 亦可应用于 $\mathrm{Cu}^{2+}$ 的检测 ${ }^{[30]}$. 例 如, 杨小峰等 ${ }^{[31]}$ 先合成了 HBT 类化合物 $\mathbf{1 6}$, 再将其与 2-(4-二乙基氨基-2-羟基苯甲酰基)-苯甲酸(17)反应，最 终可得到具有 HBT 结构的罗丹明类探针 18, 其能发出 $\mathrm{HBT}$ 的特征荧光, 且仅 $\mathrm{Cu}^{2+}$ 的加入会催化 18 发生水解、 
开环反应，使 ESIPT 型荧光机制消失，呈现出罗丹明类 化合物的特征苂光, 即苂光发射峰从 $450 \mathrm{~nm}$ 红移至 575 $\mathrm{nm}$ (Scheme 7), 故 18 可作为选择性检测 $\mathrm{Cu}^{2+}$ 的比率型 苂光探针.

电荷转移机理广泛被应用于金属离子的检测当 中 $^{[32]}$ (包括 ICT 机理 ${ }^{[20,33]}$ ). 因此, 基于电荷转移机理的 探针分子原本在溶液中能发出一定颜色的荧光, 当其与 $\mathrm{Cu}^{2+}$ 配位形成配合物后, 电荷发生转移, 溶液苂光猝灭 或者颜色改变, 籍此可以实现对 $\mathrm{Cu}^{2+}$ 的检测. 例如, Wagh 等 ${ }^{[34]}$ 设计的探针分子 19 (Scheme 8) 在体积比为 $1: 1$ 的甲醇水体系中随着 $\mathrm{Cu}^{2+}$ 的加入, 电荷从 $\mathrm{Cu}^{2+}$ 转 移到 19 上，存在显著的荧光猝灭响应.

Tung 等 ${ }^{[35]}$ 则依据炔类化合物与一价铜反应生成了 炔铜化合物 21 的原理设计并合成了用于间接检测 $\mathrm{Cu}^{2+}$
的反应型荧光探针 20 (Scheme 9). 在含有还原剂抗坏血 酸钠 $(\mathrm{NaAsc})$ 和探针 $\mathbf{2 0}$ 的水溶液中滴加 $\mathrm{Cu}^{2+}$, 苂光发生 明显的猝灭，但其它 +2 价金属离子 $\left(\right.$ 如 $\mathrm{Ni}^{2+}, \mathrm{Mn}^{2+}$, $\mathrm{Mg}^{2+}$ 等)的加入则没有引起溶液荧光的变化.

\section{3 检测铁离子}

苯并噻唑基苂光探针用于 $\mathrm{Fe}^{3+}$ 的检测，已有文献报 道 ${ }^{[36]}$, 而且一般是通过与金属离子的配位引起苂光变 化的 ${ }^{[37]}$. 例如, 刘相课题组 ${ }^{[38]}$ 通过亲核取代反应构建醚 键的方式, 将两个 HBT 结构同时接在噁二唑环的两侧, 合成了具有 4 个亚胺 $\mathrm{N}$ 配位原子的探针分子 22 (Eq. 3), 其与 $\mathrm{Fe}^{3+}$ 发生配位作用后，触发 CHEF 效应，苂光量子 产率从 0.073 提高到了 0.13 , 苂光光谱发生红移并增强 约 103 倍, 检测限为 $6.04 \times 10^{-8} \mathrm{~mol} \cdot \mathrm{L}^{-1}$.

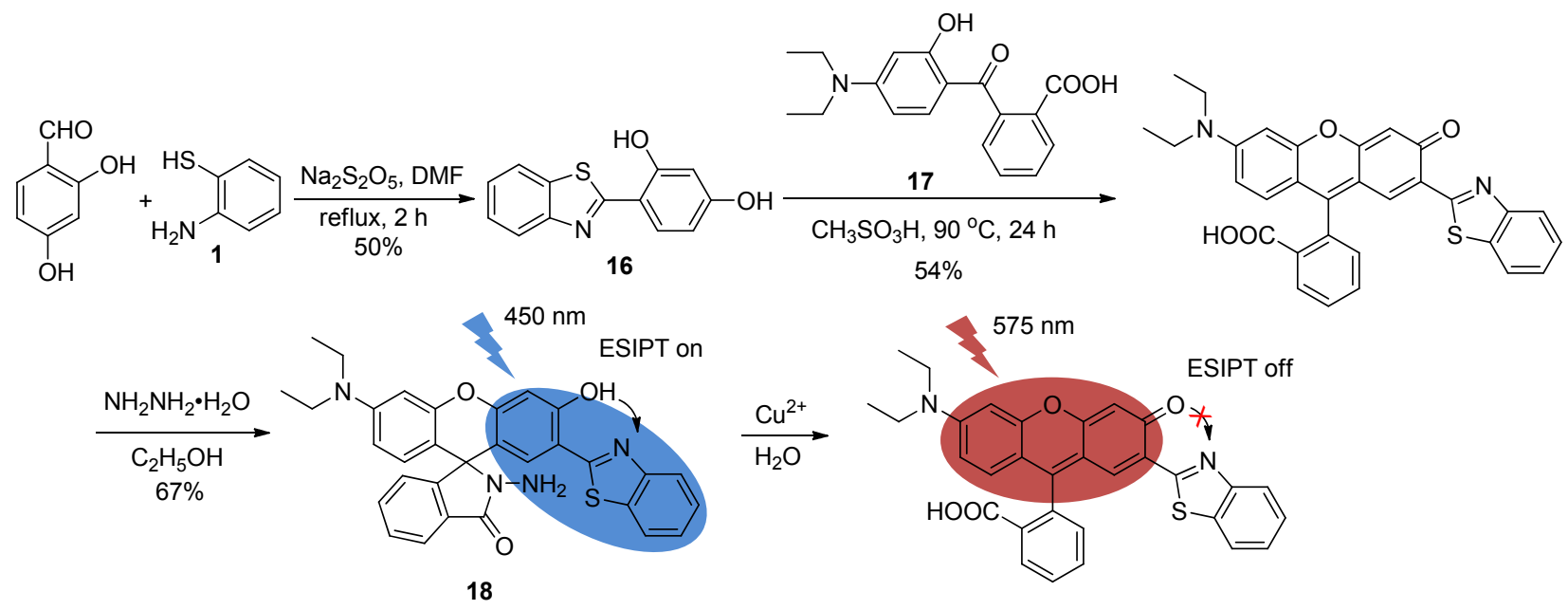

图式 7 探针 18 的合成与作用机理

Scheme 7 Synthesis of probe $\mathbf{1 8}$ and its interaction mechanism

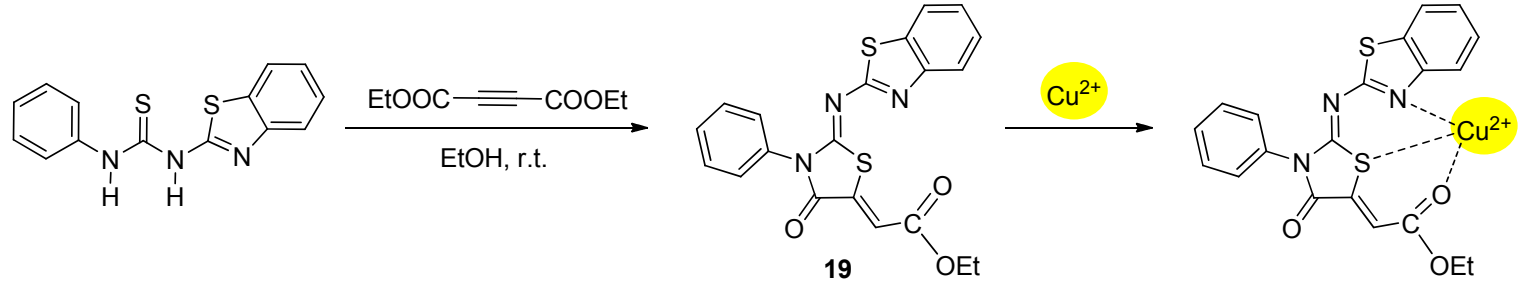

图式 8 探针 19 的合成与作用机理

Scheme 8 Synthesis of probe 19 and its interaction mechanism

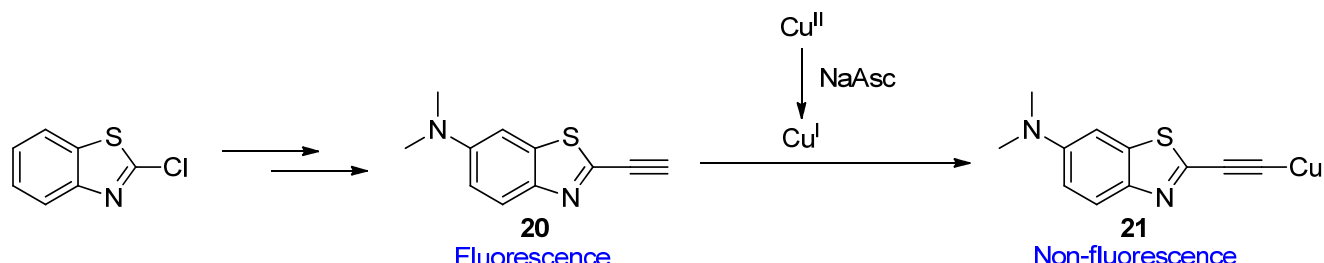

图式 9 探针 $\mathbf{2 0}$ 的合成与作用机理

Scheme 9 Synthesis of probe $\mathbf{2 0}$ and its interaction mechanism 
<smiles>Oc1ccccc1-c1nc2ccccc2s1</smiles>

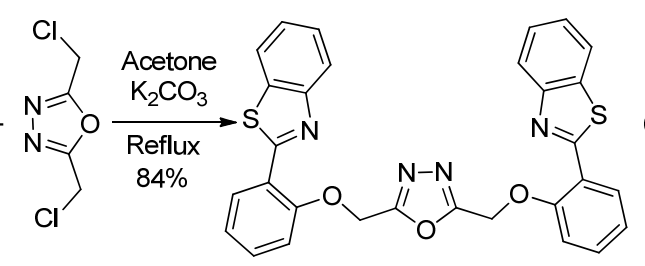

22
在检测 $\mathrm{Fe}^{3+}$ 时, 能够排除 $\mathrm{Fe}^{2+}$ 的干扰是很有意义 的. Das 等 ${ }^{[39]}$ 基于荧光共振能量转移(FRET)原理设计合 成了比率型探针分子 23 (Scheme 10). 其中, 苯并噻唑 基喹啉作为能量供体, 通过合适长度 $\left(1 \times 10^{-9} \sim 10 \times\right.$ $\left.10^{-9} \mathrm{~m}\right)$ 的桥联基团与能量受体基团罗丹明 $-6 \mathrm{G}$ 结合. 区 别于包括 $\mathrm{Fe}^{2+}$ 在内的其它生物、环境上常见的金属离子, 探针 23 仅在加入 $\mathrm{Fe}^{3+}$ 后发生荧光光谱的变化, 颜色由 蓝色转变为黄绿色, 可见光下则是由无色变为橘黄色, 故该探针能够选择性地可视化识别 $\mathrm{Fe}^{3+}$.

\section{4 检测锌离子}

苯并噻唑基苂光探针也可以检测 $\mathrm{Zn}^{2+}$, 并且也是 基于配位作用 ${ }^{[40]}$, 特别是利用多个 $\mathrm{N}$ 原子配位作用所产 生的 CHEF 效应, 这方面 Das 等 ${ }^{[41 ~ 43]}$ 做了系列工作, 合 成了 “turn on” 型 $\mathrm{Zn}^{2+}$ 苂光探针 24 26 (图 1). 无一例 外的是, 由于分子中 $\mathrm{C}=\mathrm{N}$ 容易异构化, 使这些探针有 光致电子转移(PET) 效应产生, 导致其苂光微弱, 但在 其与 $\mathrm{Zn}^{2+}$ 配位形成配合物后, $\mathrm{C}=\mathrm{N}$ 的异构化过程被抑 制, 分子结构被固定, 从而抑制了 PET 过程, 使苂光增 强. 不仅如此, 这些探针还显示出良好的选择性与纳摩 尔浓度级别的检测限.<smiles>Cc1cc(/C=N/NNc2nc3ccccc3s2)c(O)c(/C=N/Nc2nc3ccccc3s2)c1</smiles>

图 1 探针 24 26 的结构式

Figure 1 Structures of probes $\mathbf{2 4} \sim \mathbf{2 6}$

如果在功能化的苯并噻唑基团上引入长链结构, 其 与 $\mathrm{Zn}^{2+}$ 配位后可使探针分子具有自组装的能力, 有望 根据聚集诱导发光(AIE)效应检测 $\mathrm{Zn}^{2+}$ ，如宋波等 ${ }^{[44]}$ 设 计合成了含长链烷氧链的 HBT 型探针分子 27 (Scheme 11). 通过动态光散射研究与原子力显微镜观察, 发现探 针 27 本身在乙醇中不会发生聚集, 但 $\mathrm{Zn}^{2+}$ 加入后, 形 成的配合物分子发生自组装而聚集, $365 \mathrm{~nm}$ 光照下苂光 强度明显增加, 而其它十2 价金属离子 $\left(\mathrm{Pb}^{2+}, \mathrm{Co}^{2+}, \mathrm{Ni}^{2+}\right.$ 等)无此 AIE 效应，故 27 可高选择性地检测 $\mathrm{Zn}^{2+}$.

\section{5 检测其它金属阳离子}

除了应用于上述的几种常见金属离子的检测外，还 有一些文献报道了苯并噻唑基探针用于钯、铅等其它重 金属离子的检测 ${ }^{[40]}$. 例如, Kumar 等 ${ }^{[45]}$ 把 HBT 分子 4 炔 丙基化，构建了基于 $\mathrm{Pd}^{2+}$ 与炔烃发生加成反应的反应型 苯并噻唑基苂光探针 $\mathbf{2 8}$ (Scheme 12). 在探针 $\mathbf{2 8}$ 的 4-着㔯<smiles>O=Cc1ccc2cccc(O)c2n1</smiles><smiles>Nc1ccccc1S</smiles>

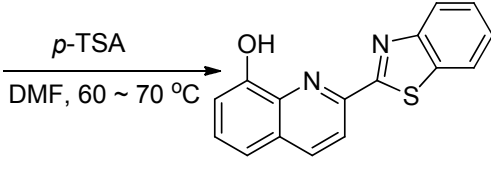
$\mathrm{K}_{2} \mathrm{CO}_{3}, \mathrm{BAB}, \mathrm{DM}$ $\mathrm{K}_{2} \mathrm{CO}_{3}$, TBAB, DMF r.t., $12 \mathrm{~h}$<smiles>CCOC(=O)COc1cccc2ccc(-c3nc4ccccc4s3)nc12</smiles><smiles>NCCNC(=O)COc1cccc2ccc(-c3nc4ccccc4s3)nc12</smiles>

rhodamine-6G

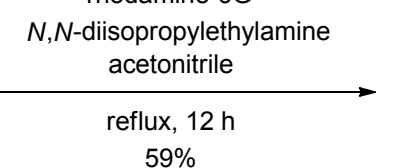
$\mathrm{H}_{2} \mathrm{~N}$<smiles>CC=CCc1ccccc1</smiles>

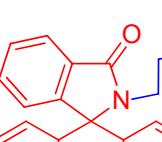<smiles>[Si]c1ccccc1</smiles><smiles>O=C(O)CC1CCCCC1</smiles>

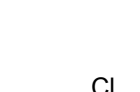<smiles>c1ccc2scnc2c1</smiles>
$\mathrm{C}$

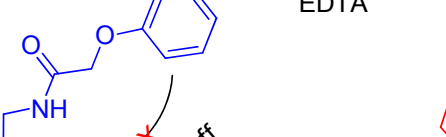

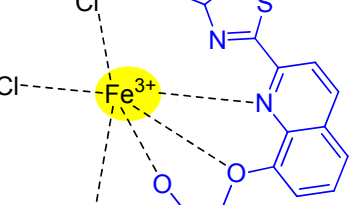<smiles>CCCCC</smiles>

图式 10 探针 23 的合成与作用机理

Scheme 10 Synthesis of probe $\mathbf{2 3}$ and its interaction mechanism 


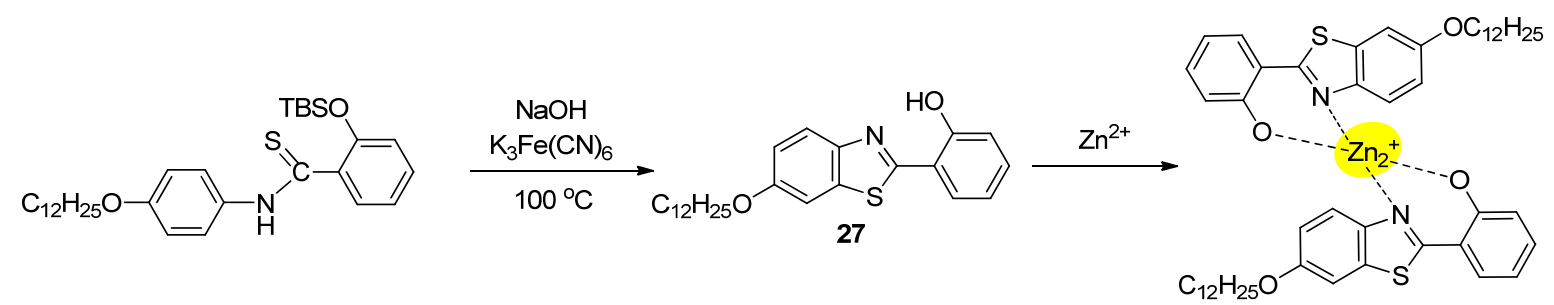

图式 11 探针 27 的合成与配合物的结构

Scheme 11 Synthesis of probe 27 and the structure of complex

乙基哌嗪乙磺酸(HEPES)缓冲溶液中, 随着氯化钯的逐 渐加入, 原本在 $372 \mathrm{~nm}$ 处的荧光发射峰逐渐减弱, 至最 终消失. 这一过程, 正是探针 28 与二价钯发生加成反 应, 生成可诱发 PET 效应的烯烃中间体 29 的过程. 但 是，经过一段时间后，水解作用的去炔丙基化，使中间 体 29 回到 HBT 分子 4 的形态, 触发 ESIPT 机制, 从而 在 $472 \mathrm{~nm}$ 处形成新的发射峰, 呈现出肉眼可见的蓝色 苂光.

铅作为一种毒性较大的重金属, 对环境和生命体的 破坏最严重. 因此, 杨晓凤课题组 ${ }^{[46]}$ 充分利用苯并噻唑 基团可同时作为配体和苂光团的双重特点，设计合成了 “turn on” 型探针 30 (Scheme 13)，其与 $\mathrm{Pb}^{2+}$ 以 $1: 2$ 配 位后，在 $585 \mathrm{~nm}$ 处的橙黄色荧光显著增强，对 $\mathrm{Pb}^{2+}$ 的检 测限可达 $2.3 \times 10^{-10} \mathrm{~mol} \cdot \mathrm{L}^{-1}$. 值得注意的是，探针 30 具有良好的细胞通透性, 使其被成功应用于活细胞内 $\mathrm{Pb}^{2+}$ 的检测.

同样地，在用于检测 $\mathrm{Ag}^{+}, \mathrm{Al}^{3+}, \mathrm{Ni}^{2+}$ 等金属离子的 苯并噻唑基探针 $31^{[47]}, \mathbf{3 2}^{[48]}, \mathbf{3 3}^{[49]}$ (图 2)中，苯并噻唑基 才的引入不仅使共轭结构得以延长，苂光性能增强，还 为探针提供了 $\mathrm{S}$ 和 $\mathrm{N}$ 这两种常见的配位原子作为配位 点, 使探针能够与不同的金属离子发生配位作用，从而 改变苂光信号，达到检测金属离子的目的.
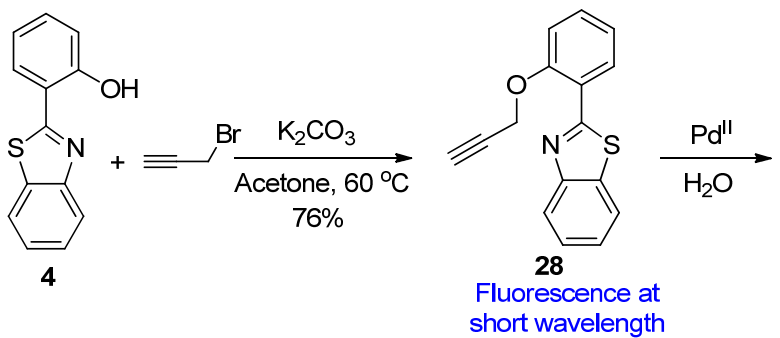
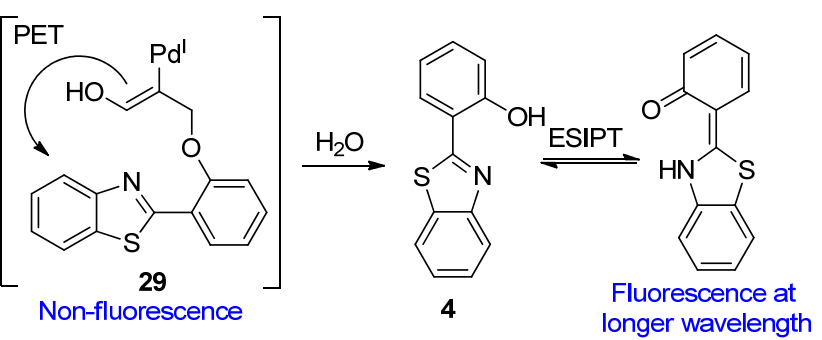

图式 12 探针 28 的合成与作用机理

Scheme 12 Synthesis of probe $\mathbf{2 8}$ and its interaction mechanism
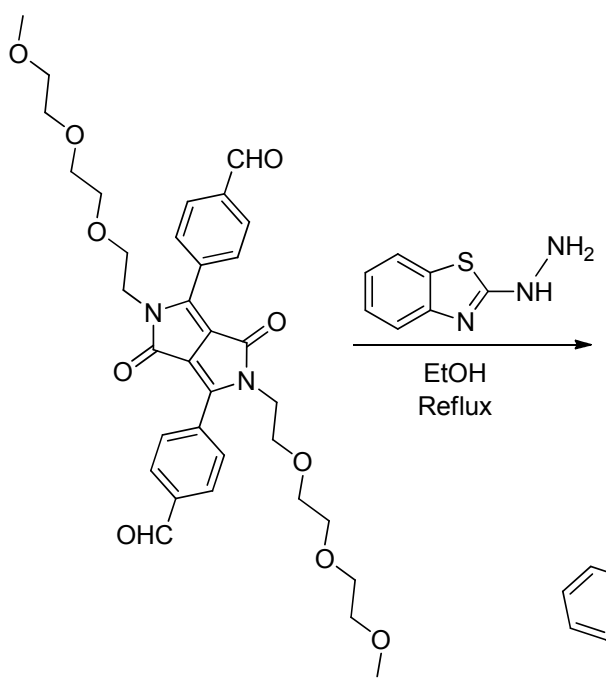

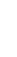

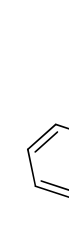<smiles>COCCOCCOCCNC=O</smiles>

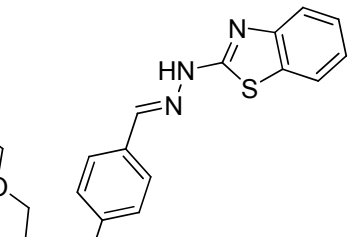

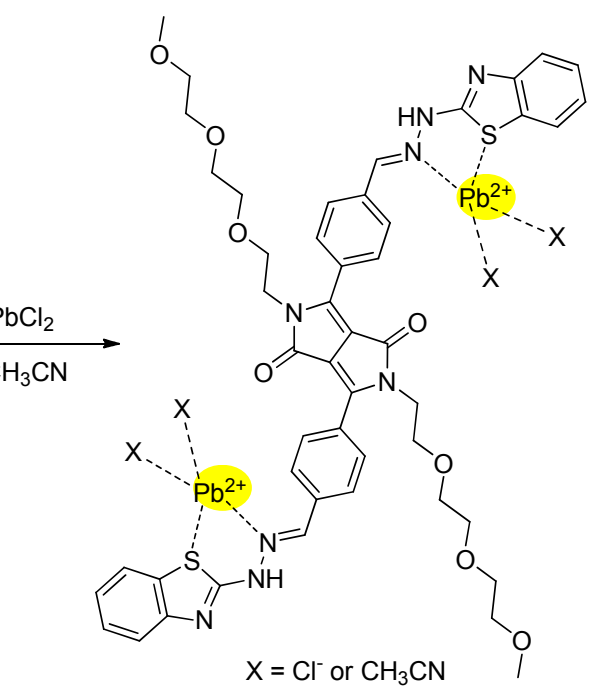

30

图式 13 探针 30 的合成与作用机理

Scheme 13 Synthesis of probe $\mathbf{3 0}$ and its interaction mechanism 
<smiles>O=C(Cc1ccc2ccccc2c1)Nc1nc2ccccc2s1</smiles><smiles>CCN(CC)c1ccc(/C=N/c2nc3ccccc3s2)c(O)c1</smiles>

32<smiles>Oc1ccc(N=Nc2cccnc2)cc1-c1nc2ccccc2s1</smiles>

图 2 探针 31 33 的结构式

Figure 2 Structures of probes $31 \sim 33$

\section{2 应用于阴离子的检测}

\section{1 检测氟离子}

氟是人体内必不可少的微量元素之一, 可以防止牙 齿疾病以及骨质疏松症 ${ }^{[50]}$. 但是, 过量的氟离子会引起 人体的氟中毒、尿石病, 甚至是癌症 ${ }^{[51]}$. 因此, 设计并 合成应用于检测氟离子的探针意义重大. 鉴于苯并噻唑 发色团良好的苂光性能 ${ }^{[52]}$, 不少含有苯并噻唑基团的 氟离子荧光探针也相继被合成, 其中包括反应型探

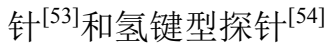

苯并噻唑基氢键型探针更为常见, 其中酚羟基上的 活泼氢是常见的 $\mathrm{F}^{-}$结合位点 ${ }^{[52]}$. 例如, Kocyigit 等 ${ }^{[55]}$ 构 建的探针 34 (Eq. 4), 其可与 $\mathrm{F}^{-}$作用形成氢键, 并逐渐 发生去质子化, 使氧原子上的电荷密度增加, 促进了 ICT 效应. 此时，最大发射峰逐渐增强并红移至 $598 \mathrm{~nm}$ 处, 呈现肉眼可见的粉色荧光. 值得注意的是, 交替加 入 $\mathrm{F}^{-}$与 $\mathrm{Ca}^{2+}$, 还可实现对 $\mathrm{F}^{-}$的可逆循环检测.

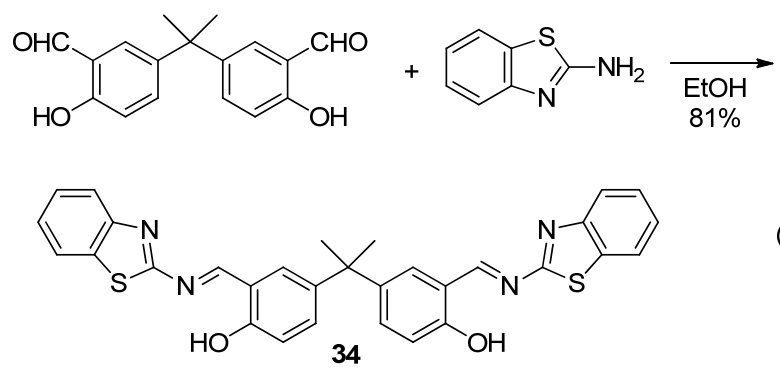

利用杂环化合物中 $\mathrm{N}-\mathrm{H}$ 作为 $\mathrm{F}^{-}$的结合位点, 是设 计氟离子荧光探针的常见手段 ${ }^{[56,57]}$, 当然也在苯并噻唑 基的荧光探针中有所体现. 例如, Razi 等 ${ }^{[58]}$ 合成的 ICT 型苂光探针 8 , 亦可用于检测 $\mathrm{F}^{-}$, 且检测限达到 $5.1 \times$ $10^{-8} \mathrm{~mol} \cdot \mathrm{L}^{-1}$. 随着 $\mathrm{F}^{-}$的加入, 探针 8 原本位于 $495 \mathrm{~nm}$ 处的强蓝绿色苂光显著猝灭, 位于 $608 \mathrm{~nm}$ 处的暗橙红 色荧光产生. 这是由于咪唑基团上的 $\mathrm{N}-\mathrm{H}$ 与 $\mathrm{F}^{-}$形成氢 键并逐渐发生去质子化, 使得原本从电子供体咪唑基团 到电子受体苯并噻唑基团的 ICT 效应进一步增强(Eq.
5). 值得一提的是, $\mathrm{CO}_{2}$ 的加入使得 $\mathbf{8}$ 与 $\mathrm{F}^{-}$的溶液恢复 到原来的蓝绿色菼光, 这也令探针 8 具备检测 $\mathrm{CO}_{2}$ 的性 能.
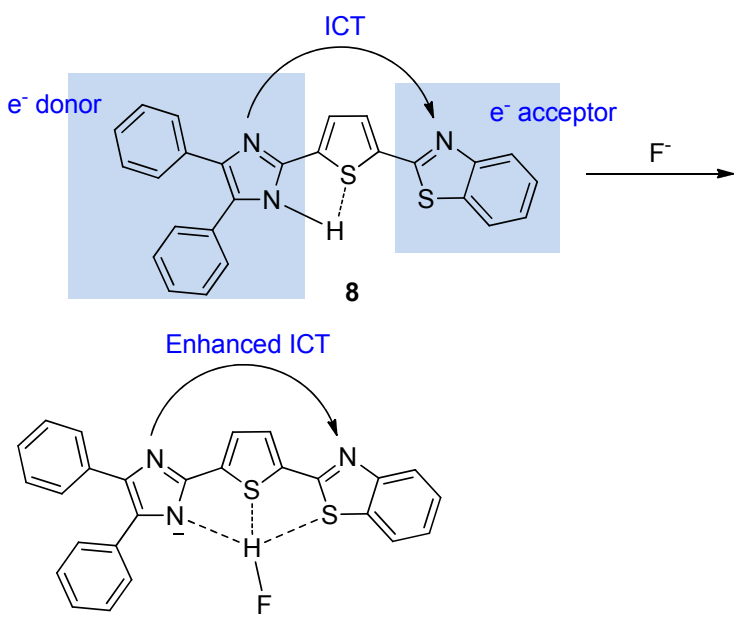

含有苯并噻唑基的反应型 $\mathrm{F}^{-}$探针，较常见的是基 于硅氧键的断裂 ${ }^{[59]}$. 例如, Goswami 等 ${ }^{[60]}$ 将 HBT 型化合 物 15 与叔丁基二甲基氯硅烷(TBDMS-Cl)反应，将羟基 保护起来，制备了探针分子 35 (Scheme 14). 由于 TBDMS 基团的保护作用，ESIPT 过程被抑制，35 在 $\mathrm{CH}_{3} \mathrm{CN}-\mathrm{HEPES}$ 混合体系中只有微弱的苂光. 当 $\mathrm{F}^{-}$加入 后，去保护作用再生成化合物 15, 使 ESIPT 过程恢复, 探针 35 原本在 $407 \mathrm{~nm}$ 处的最大荧光发射峰红移至 477 $\mathrm{nm}$ ，菼光量子产率提高至原来的 55 倍.

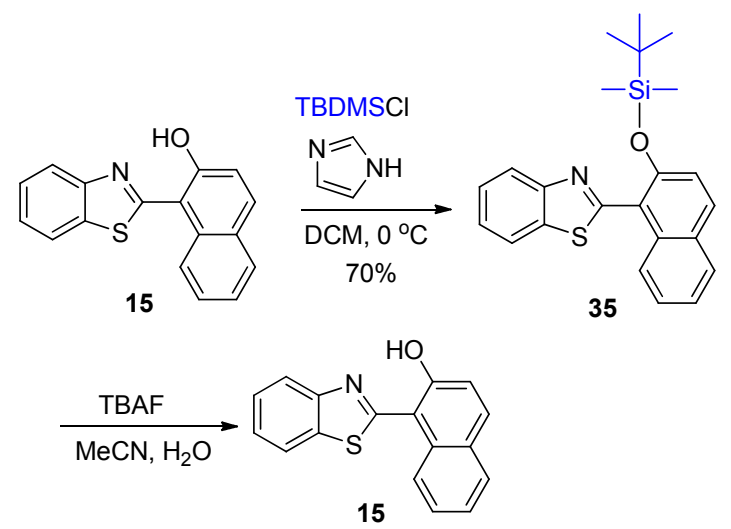

图式 14 探针 35 的合成与作用机理

Scheme 14 Synthesis of probe 35 and its interaction mechanism

\section{2 检测其它阴离子}

㲵化物是一类剧毒的物质，对环境和生命体有巨大 的危害 ${ }^{[47]}$. 因此, $\mathrm{CN}^{-}$的检测也一直受到关注. 其中, 常 见的检测原理是利用 $\mathrm{CN}^{-}$与探针结构中的双键加成生 成引起光谱变化的新化合物 ${ }^{[61]}$. 例如, Goswami 等 ${ }^{[62]}$ 合 成了探针 36 (Scheme 15), $\mathrm{CN}^{-}$可与其发生亲核加成反 应, 使电子的大离域现象被中断, 导致吸收光谱的变化 
和溶液颜色的改变. 探针 36 对 $\mathrm{AcO}^{-}, \mathrm{F}^{-}, \mathrm{Cl}^{-}$等其它阴 离子无响应，具有良好的选择性.
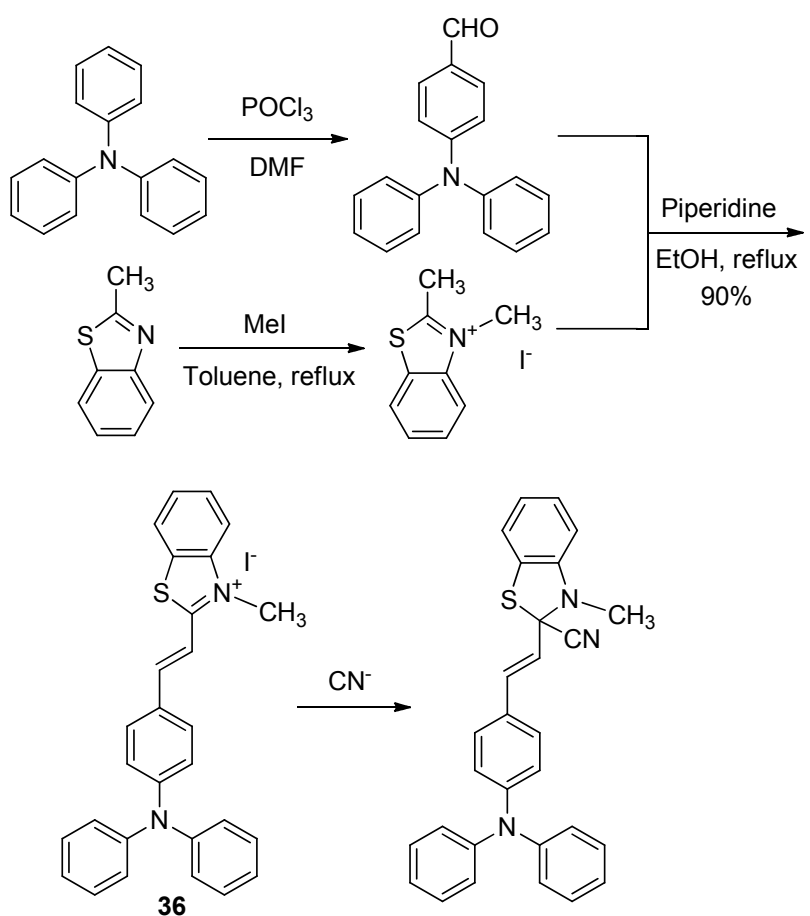

图式 15 探针 36 的合成与作用机理

Scheme 15 Synthesis of probe $\mathbf{3 6}$ and its interaction mechanism

汤立军等 ${ }^{[63]}$ 设计合成了 $\mathrm{CN}^{-}$探针 37 (Scheme 16), 其结构中含有可与 $\mathrm{CN}^{-}$作用的碳碳双键反应位点. 探针 37 原本几乎没有苂光, 但当 60 equiv. 的 $\mathrm{CN}^{-}$加入到其二 甲亚砜(DMSO)- $\mathrm{H}_{2} \mathrm{O}$ 溶液中时, 加成反应使大共轭结构 被破坏, 位于 $487 \mathrm{~nm}$ 处的苂光显著增强, 溶液颜色变为 青蓝色. 探针 37 对 $\mathrm{CN}^{-}$的检测限为 $3.94 \times 10^{-7} \mathrm{~mol} \cdot \mathrm{L}^{-1}$, 并且可用于 $\mathrm{CN}^{-}$的试纸检测.

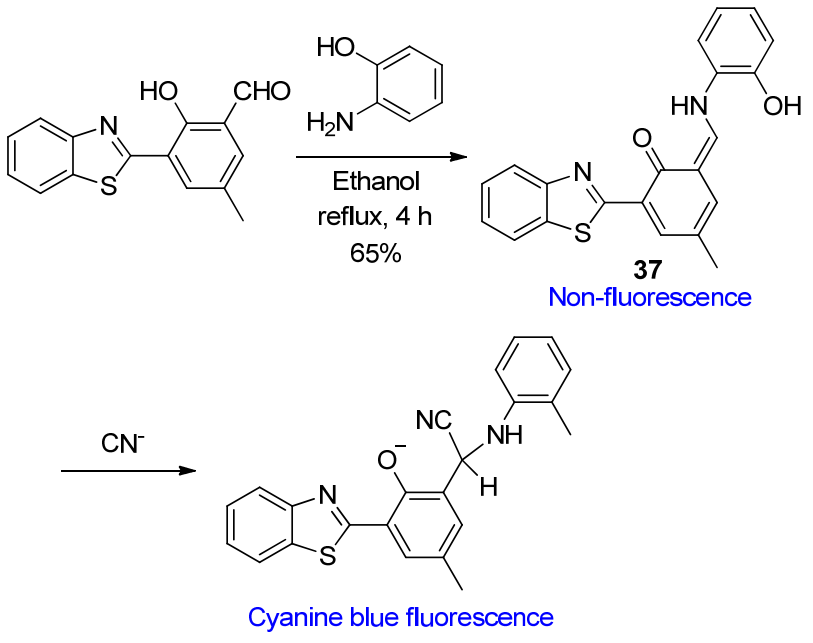

图式 16 探针 37 的合成与作用机理

Scheme 16 Synthesis of probe $\mathbf{3 7}$ and its interaction mechanism
$\mathrm{ClO}^{-}$是也一种常见的阴离子，因其高浓度情况下 对人体造成的刺激与危害，设计用于检测 $\mathrm{ClO}^{-}$的荧光 探针具有重要意义. 因此, 赵宝祥等 ${ }^{[64]}$ 合成了苯并噻唑 基肼修饰的罗丹明类探针 38 (Eq. 6). 在 38 的 $\mathrm{MeCN}-\mathrm{H}_{2} \mathrm{O}$ 溶液中滴加 $\mathrm{ClO}^{-}$后, 在 $575 \mathrm{~nm}$ 处苂光光谱 有最大发射峰产生并逐渐增强，裸眼可观察到由无色到 红色的苂光颜色变化, 对 $\mathrm{ClO}^{-}$的检测限为 $1.06 \times 10^{-9}$ $\mathrm{mol} \cdot \mathrm{L}^{-1}$.

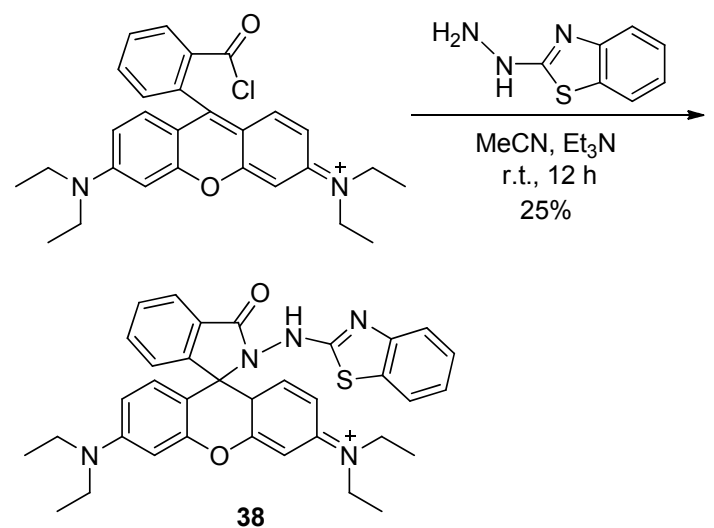

\section{3 应用于含硫化合物的检测}

\section{1 检测二氧化硫与亚硫酸盐}

二氧化硫是大气污染物之一，人体内摄入过量的二 氧化硫会导致低血压、腹泻等急性症状 ${ }^{[65]}$. 由于 $\mathrm{SO}_{2}$ 在 水中溶解后可形成 $\mathrm{H}_{2} \mathrm{SO}_{3}$, 因此多数 $\mathrm{SO}_{2}$ 苂光探针都是 把被测物 $\mathrm{SO}_{2}$ 看作 $\mathrm{SO}_{3}^{2-}$ 或 $\mathrm{HSO}_{3}^{-}$来设计合成的. 科研 工作者经常利用 $\mathrm{SO}_{3}^{2-}$ 或 $\mathrm{HSO}_{3}^{-}$能够与活性双键发生加 成反应的特点来构建二氧化硫与亚硫酸盐荧光探针 ${ }^{[66]}$.

王素华课题组 ${ }^{[67]}$ 设计合成了基于 1,4-加成反应的比 率型 $\mathrm{SO}_{2}$ 荧光探针 39, 其结构中同时含有花菁基团与 HBT 结构(Scheme 17). 对比研究表明, HBT 结构中苯并 噻唑基团不仅提升了 39 的苂光量子产率，还使发射光 谱带红移，并呈现出了第二个发射光谱带. 在 39 的 PBS 缓冲液中加入 $\mathrm{HSO}_{3}^{-}$后, 位于 $590 \mathrm{~nm}$ 处的发射峰减弱, 同时位于 $450 \mathrm{~nm}$ 处的发射峰增强, 紫外灯下苂光颜色 由玫瑰红变为蓝色. 这一荧光变化的现象归因于 $\mathrm{HSO}_{3}^{-}$ 与花菁基团中的不饱和键所发生的 1,4-加成反应破坏了 花菁染料共轭结构. 不仅如此, 生成的 1,4-加成产物在 随后 $\mathrm{H}_{2} \mathrm{O}_{2}$ 的作用下可被氧化回到原来的探针 39, 使 39 具有可逆的氧化还原活性, 能实现 $\mathrm{HSO}_{3}^{-}$的循环检测. 由于 $\mathrm{SO}_{2}$ 与 $\mathrm{HSO}_{3}^{-}$能够在水溶液中迅速达到平衡, 将溶 解了 $\mathrm{SO}_{2}$ 的乙醇加进 39 的 $\mathrm{PBS}$ 缓冲液中, 也可得到与 检测 $\mathrm{HSO}_{3}^{-}$时相似变化的苂光光谱, 即探针 39 可应用 于气态 $\mathrm{SO}_{2}$ 的检测. 

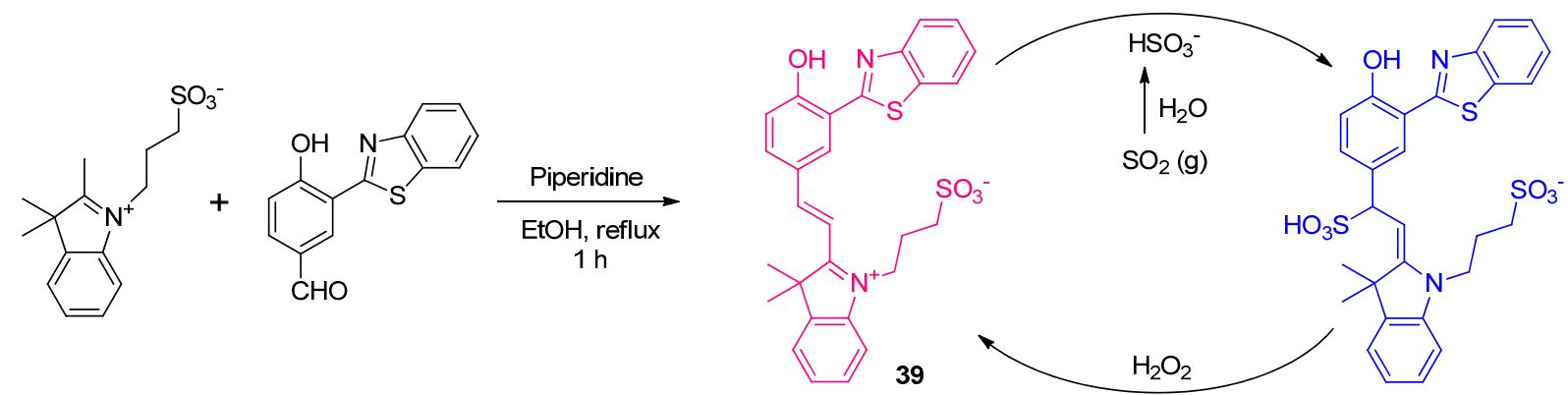

图式 17 探针 39 的合成与作用机理

Scheme 17 Synthesis of probe $\mathbf{3 9}$ and its interaction mechanism

类似地, 杨小峰课题组 ${ }^{[68]}$ 以 $\mathrm{HBT}$ 型化合物 $\mathbf{4 0}$ 为原 料, 合成了含有活性 $\mathrm{C}=\mathrm{C}$ 双键的比率型 $\mathrm{SO}_{3}^{2-}$ 苂光探 针 41 (Scheme 18). 探针 $\mathbf{4 1}$ 本身在 $578 \mathrm{~nm}$ 处有最大的 发射峰, 但仅在加入亚硫酸盐后, 荧光光谱蓝移 125 $\mathrm{nm}$, 在 $453 \mathrm{~nm}$ 处产生新的发射峰, 苂光变为蓝色. 这是 因为 $\mathrm{SO}_{3}^{2-}$ 与探针 41 的加成反应激活了 $\mathrm{HBT}$ 结构的 ESIPT 效应, 产生了 HBT 结构的特征荧光. 这种明显的 荧光颜色变化还可在人的肝星状细胞内观察到, 因此探 针 41 能实现活细胞内 $\mathrm{SO}_{3}^{2-}$ 的检测.<smiles>Oc1ccc(-c2nc3ccccc3s2)c(O)c1</smiles><smiles>CCN(CC)c1ccc(C=O)c(O)c1</smiles>

PPA, $140^{\circ} \mathrm{C}, 6 \mathrm{~h}$ $85 \%$ 40

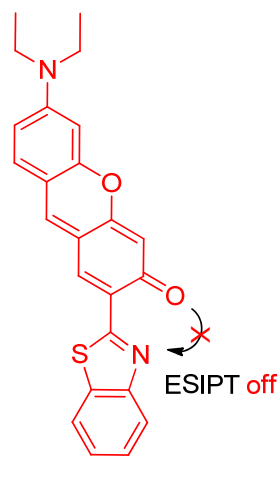

41<smiles>CCN(CC)c1ccc2c(c1)Oc1cc(O)c(-c3sc4ccccc4[n+]3CC)cc1C2S(=O)(=O)O</smiles>

图式 18 探针 41 的合成与作用机理

Scheme 18 Synthesis of probe $\mathbf{4 1}$ and its interaction mechanism

$\mathrm{C}=\mathrm{O}$ 是常见的亲核加成反应官能团, 也可籍此设 计出 $\mathrm{SO}_{2}$ 荧光探针. 例如, 宋相志课题组 ${ }^{[69]}$ 将醛基引入 HBT 型化合物中, 合成了探针 42 . 由于醛基的吸电子作 用, 探针 42 自身具有 ICT 效应. 但当其缓冲溶液中加入 $\mathrm{SO}_{3}^{2-}$ 后, 对醛基的加成反应生成了化合物 43, ICT 效应 被抑制, 仅存在 ESIPT 过程, 故荧光发射峰蓝移, 荧光 颜色在几秒钟内由黄色变为蓝色(Scheme 19), 探针 42 可实现比率型快速检测 $\mathrm{SO}_{3}^{2-}$.

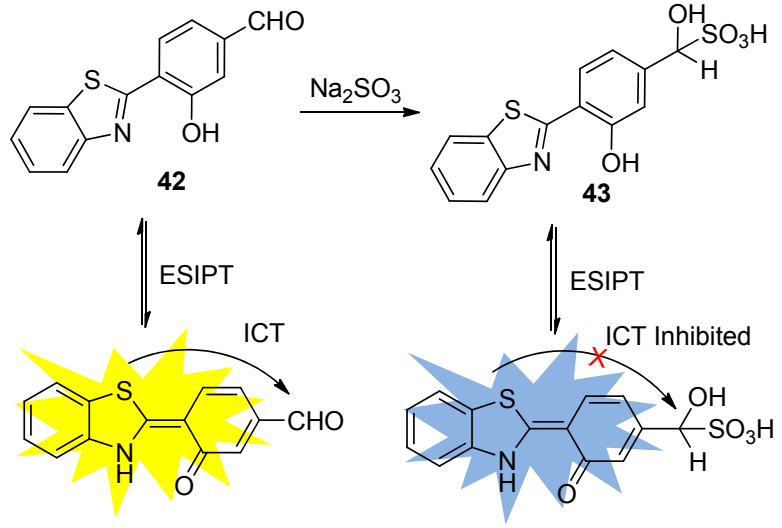

图式 19 探针 43 与 $\mathrm{SO}_{3}^{2-}$ 的作用机理 Scheme 19 Interaction mechanism of probe 43 for $\mathrm{SO}_{3}^{2-}$

\section{2 硫化氢与硫酚}

硫化氢是剧毒物质，且越来越多的证据显示阿尔茨 海默病、糖尿病、肝硬化等严重疾病的产生与人体内非 正常的 $\mathrm{H}_{2} \mathrm{~S}$ 浓度密切相关 ${ }^{[70]}$. 因此, 用于检测 $\mathrm{H}_{2} \mathrm{~S}$ 的苯 并噻唑基探针逐渐成为人们关注的焦点 ${ }^{[71]}$. 其中, 不少 探针都是基于 $\mathrm{HS}^{-}$还原叠氮基团的反应机理设计的 ${ }^{[72]}$.

郭炜课题组 ${ }^{[73]}$ 首先合成了类似 HBT 的前体化合物 44, 然后再经过 Sandmeyer 反应引入叠氮基团, 得到几 乎没有荧光的探针 $\mathbf{4 5}$ (Scheme 20). 当往 $\mathbf{4 5}$ 的 PBS 溶液 中滴加 $\mathrm{NaHS}$ (通常用作 $\mathrm{H}_{2} \mathrm{~S}$ 供体)时, $450 \mathrm{~nm}$ 处产生最大 发射峰, 并且其强度随着 NaHS 浓度增加而增加, 最终 达到原来强度的 1150 倍, 苂光量子产率由 0.0064 提升 至 0.41. 这是由于探针结构中的叠氮基团被 $\mathrm{HS}^{-}$还原为 氨基, 生成了化合物 44, 激活了 ESIPT 机制, 使苂光增 强. 不仅如此, 探针 45 仅对 $\mathrm{H}_{2} \mathrm{~S}$ 产生荧光增强的信号, 且检测限达到了 $7.8 \times 10^{-8} \mathrm{~mol} \cdot \mathrm{L}^{-1}$, 故 $\mathrm{H}_{2} \mathrm{~S}$ 探针 $\mathbf{4 5}$ 具有 很高的选择性和灵敏度.

同样地，常希俊等 ${ }^{[74]}$ 基于 HBT 型化合物 4 构建了 比率型 $\mathrm{H}_{2} \mathrm{~S}$ 探针分子 $\mathbf{4 6}$ (Scheme 21). 46 的 PBS 溶液在 $370 \mathrm{~nm}$ 处有最大的荧光发射峰, 但与 $\mathrm{H}_{2} \mathrm{~S}$ 作用后, 探针 46 中的叠氮基团被还原为氨基, 随后发生分子内 

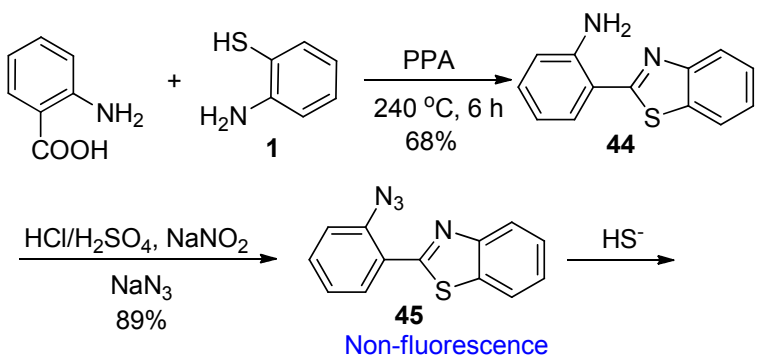

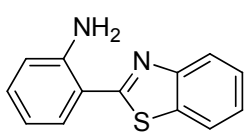

44

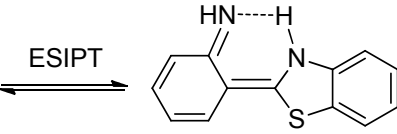

Strong fluorescence
图式 20 探针 45 的合成与作用机理

Scheme 20 Synthesis of probe $\mathbf{4 5}$ and its interaction mechanism

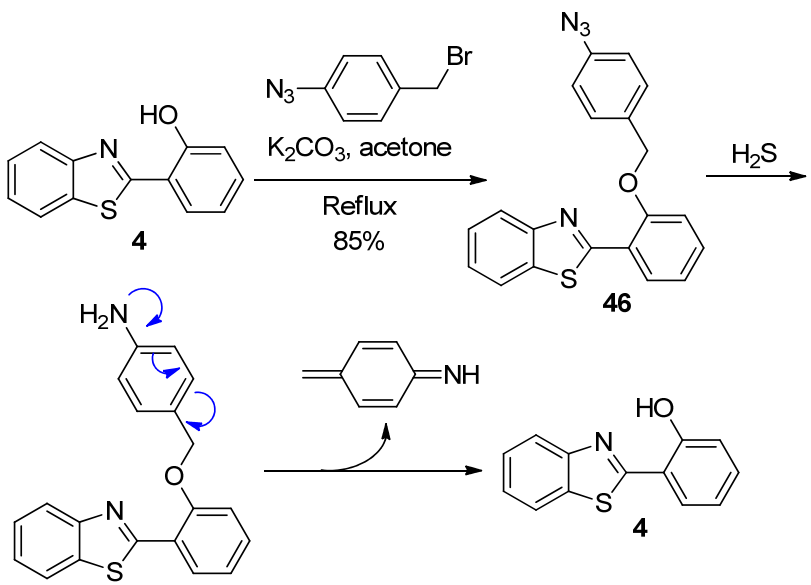

图式 21 探针 46 的合成与作用机理

Scheme 21 Synthesis of probe $\mathbf{4 6}$ and its interaction mechanism

1,6-消除，使氨基苄基离去，进而释放出 HBT 结构，导 致原来 $370 \mathrm{~nm}$ 处的发射峰强度降低, 并伴随着 $465 \mathrm{~nm}$ 处新发射峰的产生, 两处的峰值比随 $\mathrm{H}_{2} \mathrm{~S}$ 的浓度呈现良 好的线性关系. 除此之外, 探针 46 还能适应较宽 $\mathrm{pH}$ 值 范围的检测环境, 也具有较低的细胞毒性, 能成功实现 活细胞内对 $\mathrm{H}_{2} \mathrm{~S}$ 的检测. 最近, 汤立军等 ${ }^{[75]}$ 也基于该机 理设计合成了此类 $\mathrm{H}_{2} \mathrm{~S}$ 探针.

$\mathrm{HS}^{-}$还可与探针中的双键发生亲核加成反应，据此 原理设计的苯并噻唑基荧光探针也有报道 ${ }^{[76]}$. 例如, Goswami 等 ${ }^{[77]}$ 将苯并噻唑苂光团与含有活性 $\mathrm{C}=\mathrm{N}$ 双键 的花菁基团相连，合成了比率型 $\mathrm{H}_{2} \mathrm{~S}$ 苂光探针 47 (Scheme 22). 该探针与 $\mathrm{H}_{2} \mathrm{~S}$ 反应后, 裸眼可见其 $\mathrm{CH}_{3} \mathrm{CN}-\mathrm{H}_{2} \mathrm{O}$ 溶液由粉色变为黄色. 与此同时, 其苂光 光谱位于 $585 \mathrm{~nm}$ 处的最大发射峰蓝移至 $440 \mathrm{~nm}$ 处, 且 强度增加为原来的 104 倍, 原因是 $\mathrm{HS}^{-}$与 47 发生亲核加 成后, $\pi$ 共轭结构消失, 探针自身存在的 ICT 效应被终 止. 另外, 探针 47 对 $\mathrm{H}_{2} \mathrm{~S}$ 的检测具有较高选择性, 其它 阴离子或亲核试剂( $\mathrm{F}^{-}, \mathrm{HSO}_{3}^{-}, \mathrm{NH}_{3}, \mathrm{NH}_{2} \mathrm{OH}$ 等)均对 47
无响应.

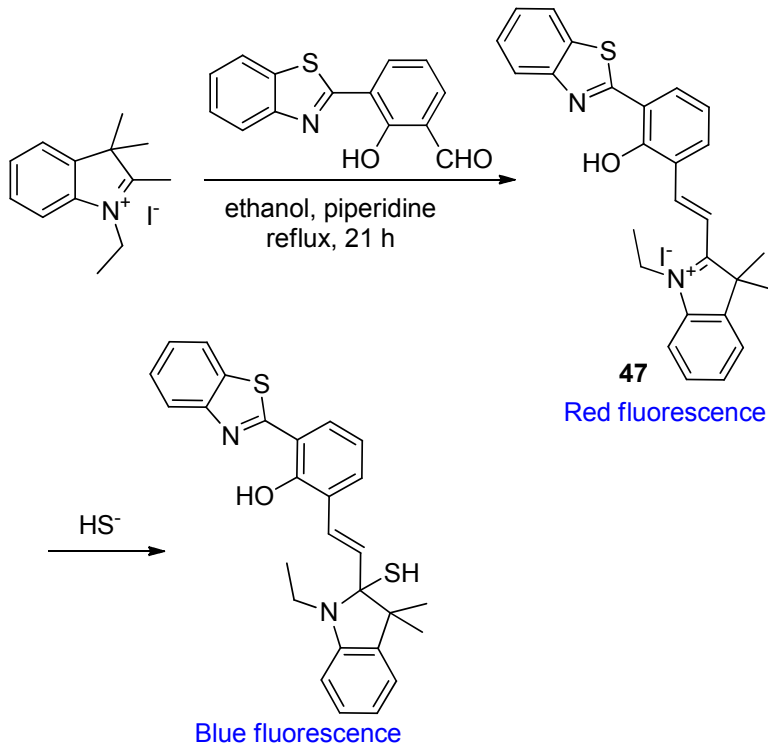

图式 22 探针 47 的合成与作用机理

Scheme 22 Synthesis of probe 47 and its interaction mechanism

不仅可以利用亲核加成反应构建苂光探针，也可以 利用 $\mathrm{HS}^{-}, \mathrm{RS}^{-}$等与探针分子发生亲核取代反应时释放 出荣光团的性质来构建苯并噻唑基苂光探针 ${ }^{[78]}$. 例如, 刘毅等 ${ }^{[79]}$ 以苯并噻唑基苯酚 48 为苂光团，与 2,4-二硝基 氟苯反应构建醚键而引入亲核反应位点，制备了荧光探 针 49 (Scheme 23). 该探针对 $\mathrm{H}_{2} \mathrm{~S}$ 不仅具有出色的选择 性，而且还能实现在活体细胞与组织内成像检测.<smiles>Cc1ccc(O)cc1</smiles><smiles>Oc1ccc(-c2nc3ccccc3s2)cc1</smiles><smiles>O=[N+]([O-])c1ccc(OCC(F)(F)F)c([N+](=O)[O-])c1</smiles>
$80^{\circ} \mathrm{C}, 6 \sim 8 \mathrm{~h}$ $85 \%$<smiles>O=[N+]([O-])c1ccc(Oc2ccc(-c3nc4ccccc4s3)cc2)c([N+](=O)[O-])c1</smiles>

48<smiles>O=[N+]([O-])c1ccc(S)c([N+](=O)[O-])c1</smiles>

49

图式 23 探针 49 的合成与作用机理

Scheme 23 Synthesis of probe 49 and its interaction mechanism

类似的, $\mathrm{RS}^{-}$的亲核取代作用机理还常见于硫酚探 针设计中 ${ }^{[80]}$. 例如, 山东大学李敏勇等 ${ }^{[81]}$ 在 HBT 结构 上引入 2,4-二硝基苯磺酰基(DNs)，制备了探针 $\mathbf{5 0}$ 
(Scheme 24). 在 $\mathbf{5 0}$ 的 DMF-PBS 体系中加入苯硫酚后, 苂光光谱在 $450 \mathrm{~nm}$ 处出现最大发射峰并逐渐增强. 这 是由于探针 50 与苯硫酚发生了亲核取代反应, 释放了 HBT 荧光团所致.
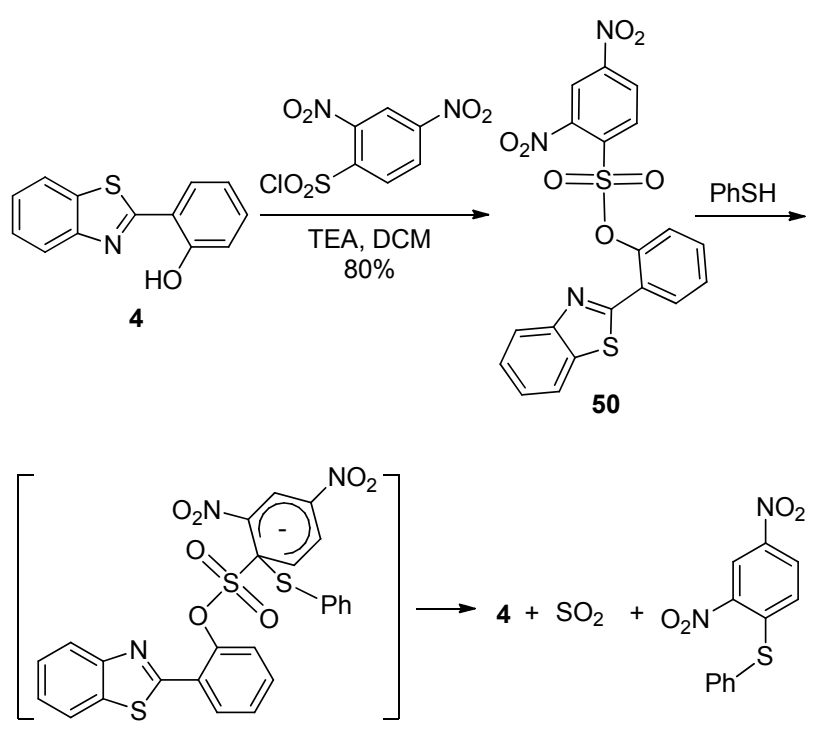

图式 24 探针 $\mathbf{5 0}$ 的合成与作用机理

Scheme 24 Synthesis of probe $\mathbf{5 0}$ and its interaction mechanism

在国外，Talukdar 等 ${ }^{[82]}$ 以亚氨基香豆素和苯并噻唑 相连构建荧光团 51, 在此基础上引入吸电子的 DNs 作 为反应位点, 设计了苂光探针 $\mathbf{5 2}$. 当 $\mathrm{PhS}^{-}$亲核进攻 $\mathbf{5 2}$ 时，其与 DNs 基团作用后释放出苂光团结构 $\mathbf{5 1}$ (Scheme
25), 而硫醇则无 “off-on” 荧光响应，故探针 $\mathbf{5 2}$ 可实现 对硫酚的专一检测. 与不含苯并噻唑基的类似探针相 比，苯并噻唑基团的引入有利于苂光团具有更强的苂光 强度和更长的荧光波长.

\section{3 检测生物琉基化合物}

苯并噻唑类分子作为探针应用于生物分子的检测 也有报道 ${ }^{[83 \sim 85]}$. 其中, 由于半胱氨酸(Cys)、谷胱甘肽 (GSH)等生物巯基化合物在生化途径中扮演着重要的角 色，故对其体内检测很受关注 ${ }^{[86]}$, 并且通常是基于上述 的 $\mathrm{RS}^{-}$亲核取代检测机理设计的苯并噻唑基探针 ${ }^{[87]}$.

例如, 叶勇课题组 ${ }^{[88]}$ 设计合成了系列苯并噻唑基 荧光探针 53 055 (Scheme 26). 其中, 具有两个反应中 心的探针 55 与 Cys 类化合物作用时产生的荧光最强. 但 是, 与前面的 “off-on” 探针不同的是, 这些化合物中苯 并噻唑基团的作用是充当了生物颈基化合物的载体.

将酚羟基形成的酯键作为反应位点，是构建生物分 子探针的重要方法之一 ${ }^{[89,90]}$. 例如, 杨小峰课题组 ${ }^{[91]}$ 基 于 HBT 结构化合物 $\mathbf{5 6}$ 设计合成了丙烯酸酯类探针 $\mathbf{5 7}$, 当其遇到 Cys、同型半胱氨酸(Hcy)等含颈基的氨基酸时, 先发生加成反应生成化合物 $\mathbf{5 8}$, 接着 $\mathbf{5 8}$ 中的氨基进行 分子内的环合反应生成了化合物 $\mathbf{5 9}$, 同时释放出 $\mathbf{5 6}$ 而 启动了 ESIPT 效应，使 $\mathbf{5 8}$ 在 $377 \mathrm{~nm}$ 处特征荧光红移至 $487 \mathrm{~nm}$ 处(Scheme 27). 由于 Cys, Hcy 生成的内酰胺 59 的结构与动力学过程不同, 使二者的苂光变化过程有明 显的速度差异，故 57 可同时高效地鉴别 Cys 和 Hcy.<smiles>CCN(CC)c1ccc2cc(-c3nc4ccccc4s3)c(=N)oc2c1</smiles>

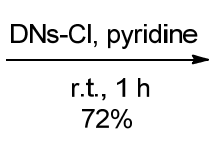
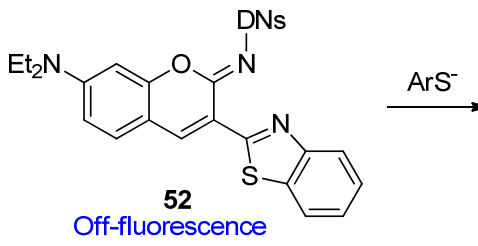

图式 25 探针 52 的合成与作用机理

Scheme 25 Synthesis of probe $\mathbf{5 2}$ and its interaction mechanism

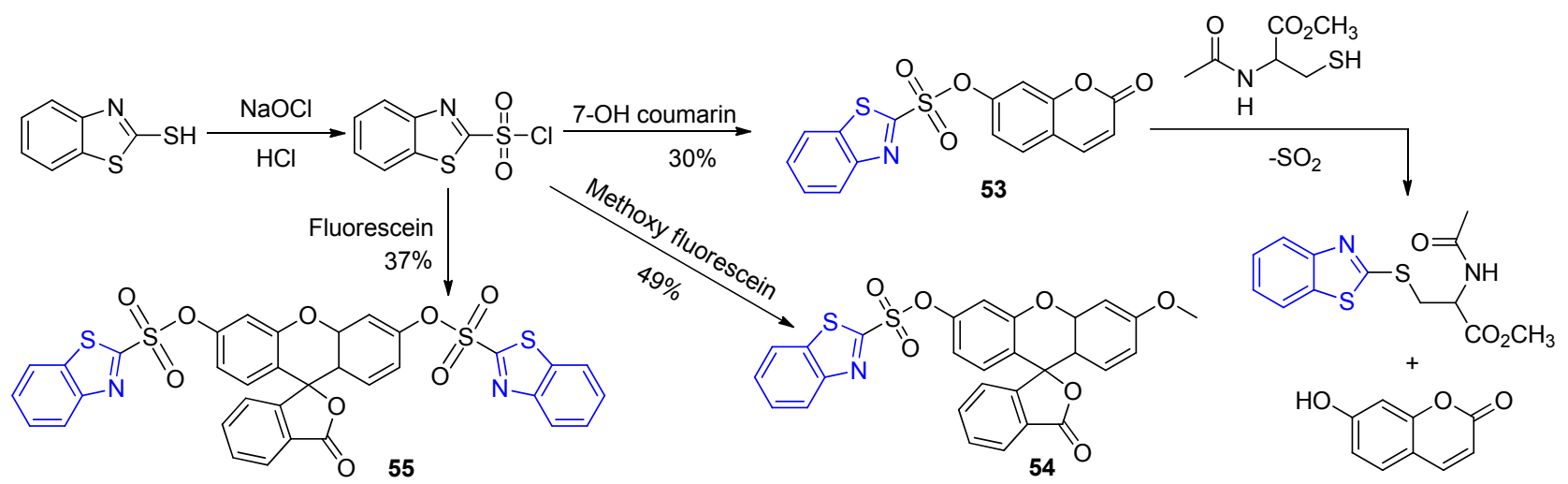

图式 26 探针 53 55 的合成与作用机理

Scheme 26 Synthesis of probes 53 55 and their interaction mechanism 
<smiles>C=CC(=O)Oc1c(OC)cccc1-c1nc2ccccc2s1</smiles>

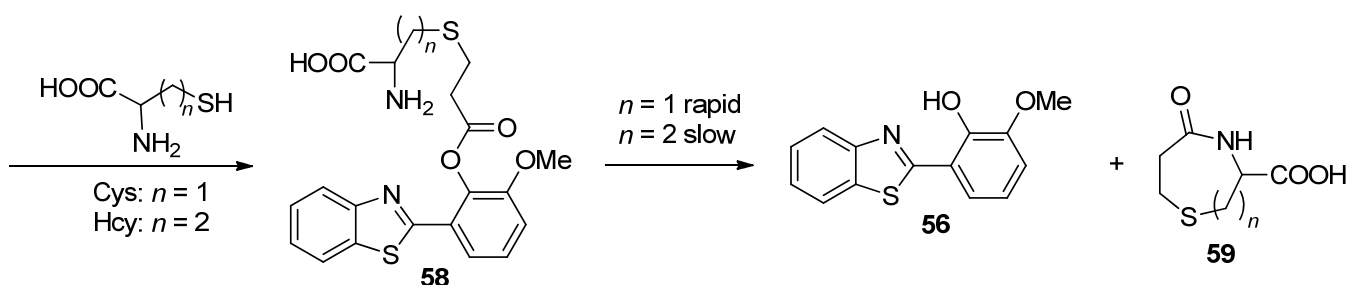

图式 27 探针 57 的合成与作用机理

Scheme 27 Synthesis of probe $\mathbf{5 7}$ and its interaction mechanism

Churchill 等 ${ }^{[92]}$ 利用类似的检测机理, 以不同的合成路 线, 也构建了 Cys 荧光探针 60 (Scheme 28).

利用 Cys (Hcy)这种含琉基的氨基酸特殊的胺解作 用, 也可构建能用于选择性区别 GSH 与 Cys (Hcy)的苯 并噻唑基苂光探针. 例如, 杨小峰等 ${ }^{[93]}$ 基于罗丹明类结 构合成了苂光探针 62 (Scheme 29), 其与 GSH, Cys, Hcy
均能发生交换反应，对硝基硫酚 61 离去后, PET 抑制效 应被解除. 对 GSH 检测时，在 $587 \mathrm{~nm}$ 处有荧光最大发 射峰. 但是, 对 Cys, Hcy 检测时由于分子内胺解、环化 反应的发生而启动了 ESIPT 效应，最大发射峰红移至 $454 \mathrm{~nm}$ 处. 这两种明显不同的苂光响应令 62 能有效地 鉴别 GSH 与 Cys(包括 Hcy).<smiles>Oc1ccccc1-c1nc2ccccc2s1</smiles>

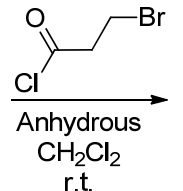
r.t.

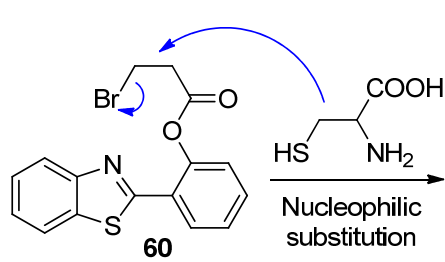<smiles>O=C(O)C1CCSCC(C(=O)Oc2ccccc2-c2nc3ccccc3s2)CNC1</smiles><smiles>CCCCCCCCCCC[C@@H]1SCCC(=O)NC1C(=O)O</smiles>

图式 28 探针 60 的合成与作用机理

Scheme 28 Synthesis of probe $\mathbf{6 0}$ and its interaction mechanism

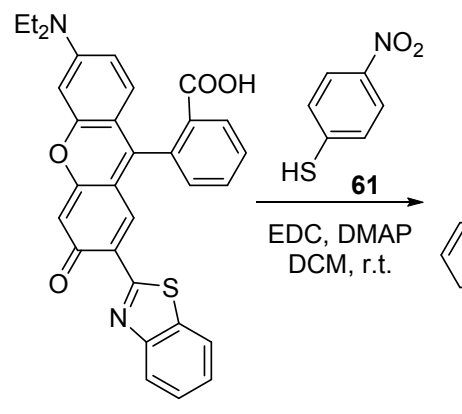

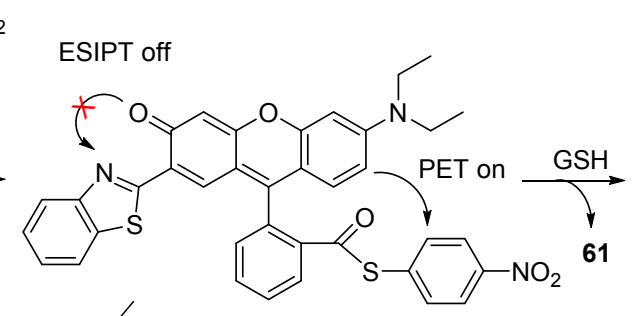

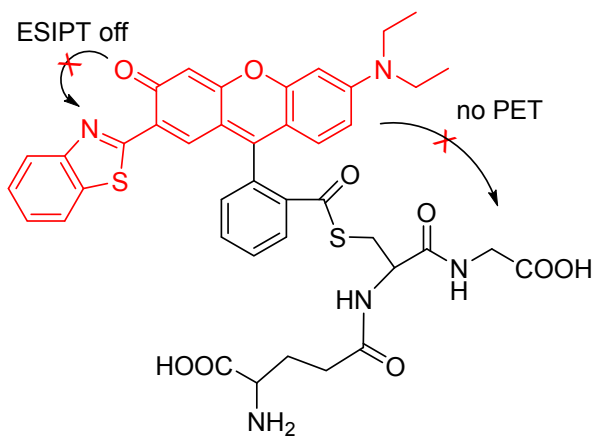

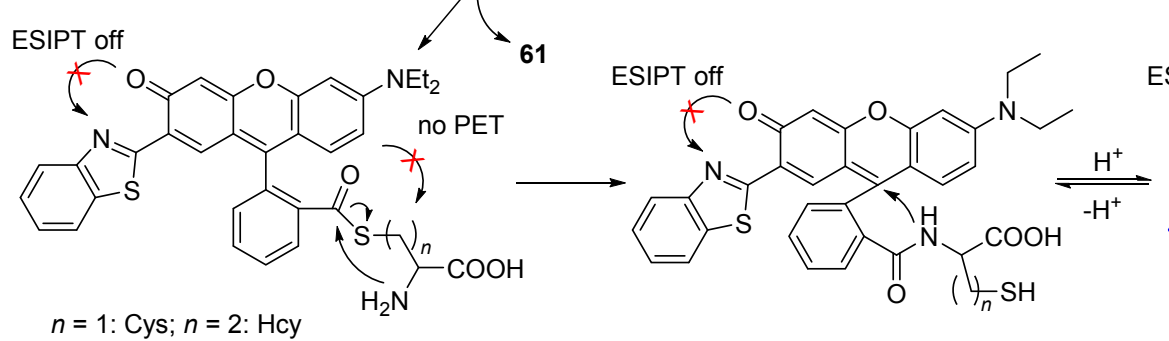<smiles></smiles>

图式 29 探针 62 的合成与作用机理

Scheme 29 Synthesis of probe $\mathbf{6 2}$ and its interaction mechanism 


\section{4 应用于其它物种的检测}

除上述的各种应用外, 苯并噻唑基荧光探针还可以 检测其它物种 ${ }^{[36]}$. 例如, Goswami 等 ${ }^{[94]}$ 设计合成了用于 检测肼类化合物的苯并噻唑基荧光探针 63 , 其检测机 理也是基于亲核取代启动的分子内环化与消去反应, 最 终导致 HBT 苂光团释放而产生比率型苂光变化(Scheme 30).

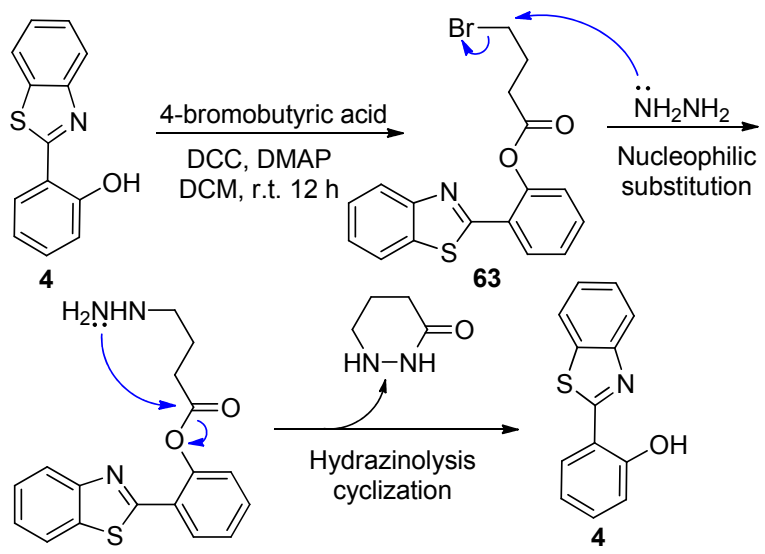

图式 30 探针 63 的合成与作用机理

Scheme 30 Synthesis of probe $\mathbf{6 3}$ and its interaction mechanism

利用苯并噻唑基荧光探针检测 $\mathrm{pH}$ 的报道也相对较 多 ${ }^{[95]}$. 其中, 这些探针通常具有比苯并噻唑更大的共轭 结构 ${ }^{[96]}$. 利用 2-甲基苯并噻唑与芳香醛的缩合反应, 是 延长 $\pi$ 共轭结构获得更大的共轭体系的方法之一 ${ }^{[97]}$, 如 比率型 $\mathrm{pH}$ 荧光探针 64 的合成(Scheme 31) ${ }^{[98]}$. 随着乙腈/ 水 $(V: V=1: 1)$ 体系 $\mathrm{pH}$ 值从 7 到 1 逐渐减小, 64 的最 大荧光发射峰从 $496 \mathrm{~nm}$ 处红移至 $583 \mathrm{~nm}$, 苂光颜色由 蓝色变为橙色. 在该过程中, 苯并噻唑基团上的 $\mathrm{N}$ 原子 发生了质子化, 导致从咔唑基团到苯并噻唑基团的 ICT 效应增强, 由此产生苂光波长红移的现象. 除此以外, 64 还成功实现了大肠杆菌中 $\mathrm{pH}$ 成像, 证实了其在强酸 性的微生态环境中可有效检测 $\mathrm{pH}^{[98]}$.
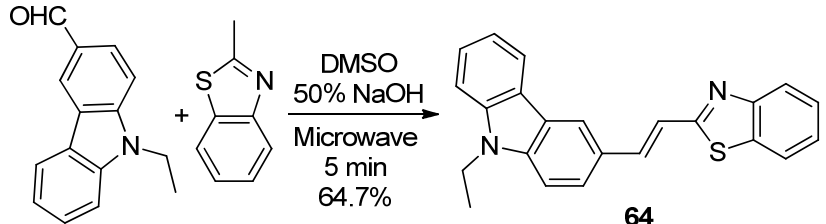

Enhanced ICT

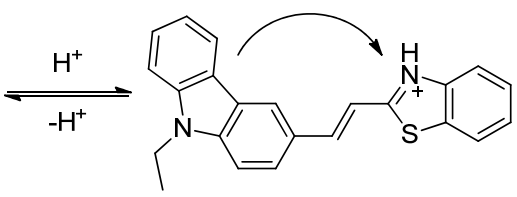

图式 31 探针 64 的合成与作用机理

Scheme 31 Synthesis of probe 64 and its interaction mechanism

事实上，苯并噻唑基团上的 $\mathrm{N}$ 原子作为质子化位 点, 是大多数苯并噻唑基 $\mathrm{pH}$ 苂光探针的作用机理 ${ }^{[99]}$. 王素华课题组 ${ }^{[100]}$ 基于 HBT 结构化合物 65 与丙酮的缩 合反应制备的探针 66 (Scheme 32), 其在酸性环境下由 于苯并噻唑上 $\mathrm{N}$ 的质子化作用, ICT 效应增强, 荧光光 谱中原本在中性环境下位于 $515 \mathrm{~nm}$ 的最大发射波长红 移至 $565 \mathrm{~nm}$ 处，且苂光量子产率也从 0.063 提高至 0.25 .

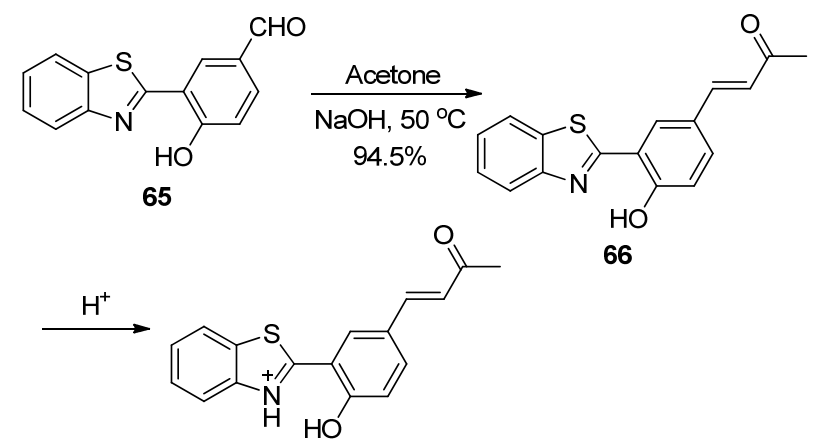

图式 32 探针 66 的合成与作用机理

Scheme 32 Synthesis of probe $\mathbf{6 6}$ and its interaction mechanism

Saha 等 ${ }^{[101]}$ 对缩合反应得到的反-2-(4-二甲胺基苯乙 烯基)苯并噻唑探针分子 67 (Scheme 33) 有较多的研究,

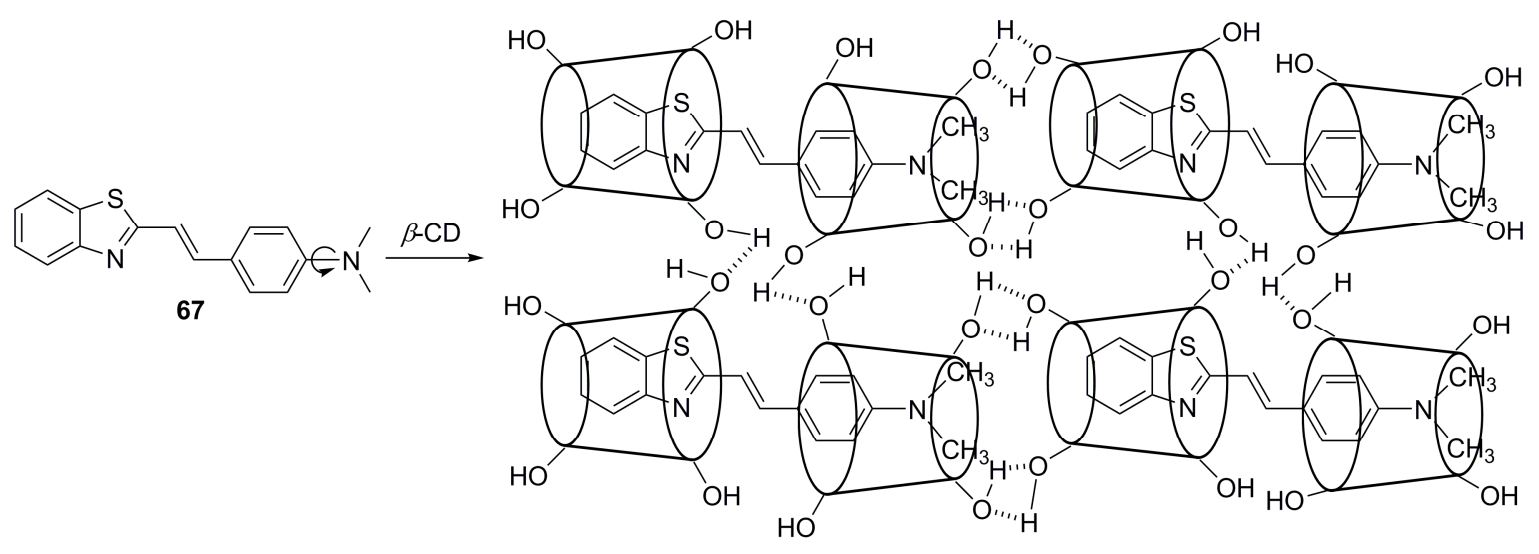

图式 33 探针 67 的作用机理

Scheme 33 Interaction mechanism of probe 67 
其可应用于多方面的检测. 由于结构中存在可自由旋转 的二甲胺基, 探针 67 自身的荧光较弱. 但在其甲醇溶液 中加入 $2.5 \mathrm{mmol} \cdot \mathrm{L}^{-1}$ 的 $\beta$-环糊精 $(\beta-\mathrm{CD})$ 后, 苂光增强并 达到最大值. 这是因为 $\beta$ - $\mathrm{CD}$ 与 67 的自组装作用形成了 杆状的纳米管(Scheme 33), 从而阻止了二甲胺基的自 由旋转 ${ }^{[102]}$. 利用类似的原理, 探针 67 还可实现对 $\mathrm{DNA}^{[103]}$ 和 Brij 表面活性剂[104]等的苂光检测.

同样基于限制自由旋转的荧光增强机制, 唐本忠课 题组 ${ }^{[105]}$ 构建了亲水性的阳离子型探针 68 (Scheme 34). 作为结构改造后 HBT 型化合物, 68 遇到阴离子表面活 性剂十二烷基苯磺酸钠后, 通过协同的静电与疏水作用 在水中形成阴阳离子聚集物, 聚集物内分子间堆积有效 地限制了 68 分子内运动, 使被抑制的 ESIPT 机制得以 恢复, 产生 AIE 效应, 故荧光光谱在 $510 \mathrm{~nm}$ 处产生最大 发射峰，苂光量子产率从 0.002 提高至 0.5 .<smiles>O=Cc1cc(CCl)ccc1O</smiles><smiles>CCCCCCCN(C)C</smiles><smiles>CN(Cc1ccc(O)c(C=O)c1)[N+](Cl)(Cl)Cl</smiles>

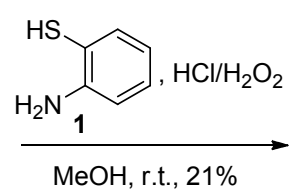<smiles>CC(C)C[N+](C)(Cl)Cc1ccc(O)c(-c2nc3ccccc3s2)c1</smiles>

图式 34 探针 68 的合成

Scheme 34 Synthesis of probe $\mathbf{6 8}$

对 HBT 型化合物进行结构修饰，也可合成检测其 它物种的苂光探针. 例如, 基于 HBT 型化合物 $\mathbf{4}$, 通过 引入含磷基团, 还可以构建用于检测超氧化物 ${ }^{[106]}$ 、硝酰 基化合物 ${ }^{[107]}$ 等的苯并噻唑基苂光探针，如化合物 69 和 70 (Scheme 35), 它们的苂光检测机制都是经过反应最 终释放出 HBT 荧光团.

\section{5 结束语}

由于苂光探针发展迅速, 针对不同检测对象的探针 每年大量涌现 ${ }^{[108]}$, 苯并噻唑基苂光探针也渗透到了金 属阳离子、阴离子、含硫化合物、生物分子等检测的领 域. 这归结于苯并噻唑类化合物以下几个特点: (1)合成 简便、原料易得; (2)具有大共轭体系, 荧光性能突出, 常 被视为荧光团引入探针结构; (3)结构中含杂原子, 能提 供与被测物结合的作用位点; (4)细胞毒性小, 能够广泛 应用于活细胞内检测. 总之, 苯并噻唑基荧光探针顺应 了当今苂光探针绿色合成、性能优良、适用范围广的发
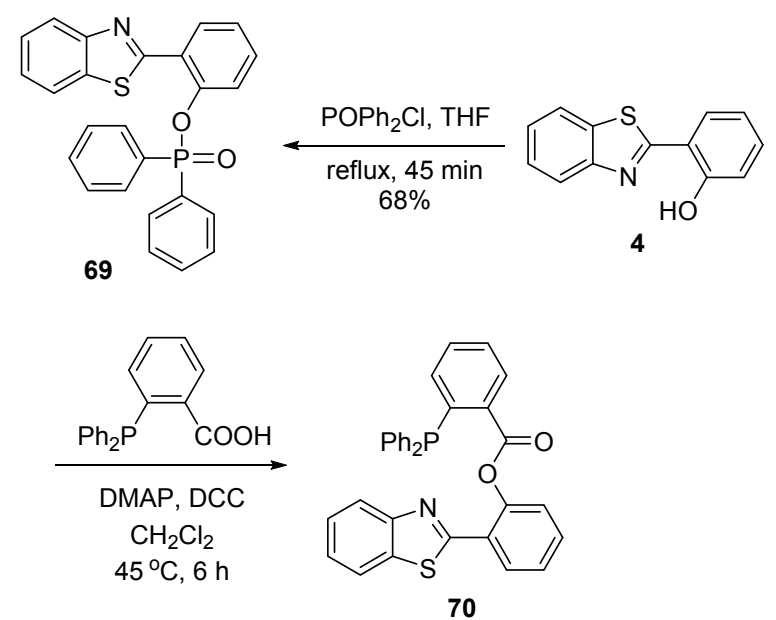

图式 35 探针 69,70 的合成

Scheme 35 Synthesis of probes 69 and 70

展趋势, 应用前景十分广阔.

展望未来, 尽管目前在生物医学和生命科学等前沿 学科的研究领域已有一些具有较好检测效果的苯并噻 唑基荧光探针被设计、合成出来 ${ }^{[109 \sim 111]}$, 但在公共安全 与环境保护等层面, 针对三硝基苯酚(PA)、三硝基甲苯 (TNT)等硝基芳香爆炸物的苂光检测 ${ }^{[112]}$, 目前仍鲜有苯 并噻唑基荧光探针的报道 ${ }^{[36]}$. 同时, 对多种金属离子同 时检测的报道也不多 ${ }^{[11,20,34,40]}$. 因此，把握苯并噻唑的 结构特点与优势, 进一步指导设计、合成应用于更多不 同检测对象苂光探针, 特别是能够进行同时检测, 仍是 极富挑战但有章可循的研究工作.

\section{References}

[1] Rouf, A.; Tanyeli, C. Eur. J. Med. Chem. 2015, 97, 911.

[2] Adzal, O.; Akhtar, M. S.; Kumar, S.; Ali, M. R.; Jaggi, M.; Bawa, S. Eur. J. Med. Chem. 2016, 121, 318.

[3] Kamal, A.; Syed, M. A. H.; Mohammed, S. M. Expert Opin. Ther. Pat. 2015, 25, 335.

[4] Zhao, L. X.; Wang, X. Y.; Li, X. D.; Zhang, W. J.; Liu, X. H.; Zhu, Y. J.; Wang, H.-Q.; Fang, J. F. Sol. Energy Mater. Sol. Cells 2016, 157,79 .

[5] Abou-Zied, O. K. RSC Adv. 2013, 3, 8747.

[6] Goswami, S.; Das, A. K.; Aich, K.; Manna, A. Tetrahedron Lett. 2013, 54, 4215.

[7] Guan, L.; Li, A. Y.; Song, Y. Y.; Yan, M. Q.; Gao, D. F.; Zhang, X. H.; Li, B.; Wang, L. Y. J. Org. Chem. 2016, 81, 6303.

[8] Kartha, K. K.; Sandeep, A.; Prave Liu, D.; Ren, H. C.; Deng, L. J.; Zhang, T. ACS Appl. Mater. Interfaces 2013, 5, 4937.

[9] Carrington, S. J.; Chakraborty, I.; Bernard, J. M. L.; Mascharak, P. K. Inorg. Chem. 2016, 55, 7852.

[10] Aich, K.; Goswami, S.; Das, S.; Mukhopadhyay, C. D.; Quah, C. K.; Fun, H.-K. Inorg. Chem. 2015, 54, 7309.

[11] Hou, S. H.; Qu, Z. G.; Zhong, K. L.; Bian, Y. J.; Tang, L. J. Chin. J. Org. Chem. 2016, 36, 768 (in Chinese).

(侯淑华, 曲忠国, 钟克利, 边延江, 汤立军, 有机化学, 2016, 36, 768.)

[12] Lee, J. H.; Lee, J. H.; Kim, S. K.; Jung, J. H. Supramol. Chem. 2015, 27, 690 .

[13] Maity, S. B.; Bharadwaj, P. K. J. Lumin. 2015, 161, 76.

[14] Ju, H.; Chang, D. J.; Kim, S.; Ryu, H.; Lee, E.; Park, I.-H.; Jung, J. 
H.; Ikeda, M.; Habata, Y.; Lee, S. S. Inorg. Chem. 2016, 55, 7448.

[15] Sahana, S.; Mishra, G.; Sivakumar, S.; Bharadwaj, P. K. Dalton Trans. 2015, 44, 20139.

[16] Bae, J.-S.; Gwon, S.-Y.; Son, Y.-A.; Kim, S.-H. Dyes Pigm. 2009, $83,324$.

[17] Singh, A.; Singh, A.; Singh, N.; Jang, D. O. Tetrahedron 2016, 72, 3535 .

[18] Erdemir, S.; Malkondu, S.; Alici, O. Color. Technol. 2015, 131, 32.

[19] Mei, Q. B.; Tian, R. Q.; Shi, Y. J.; Hua, Q. F.; Chen, C.; Tong, B. H. New J. Chem. 2016, 40, 2333.

[20] Razi, S. S.; Ali, R.; Gupta, R. C.; Dwivedi, S. K.; Shama, G.; Koch, B.; Misra, A. J. Photochem. Photobiol. A 2016, 324, 106

[21] Gu, B.; Huang, L. Y.; Su, W.; Duan, X. L.; Li, H. T.; Yao, S. Z. Anal. Chim. Acta 2017, 954, 97.

[22] Zhang, D.; Liu, J. H.; Yin, H. Y.; Wang, H. Q.; Li, S. F.; Wang, M.; Li, M.; Zhou, L.; Zhang, J. F. J. Fluoresc. 2016, 26, 1367.

[23] Santra, M.; Roy, B.; Ahn, K. H. Org. Lett. 2011, 13, 3422.

[24] Luo, W. F.; Jiang, H. E.; Zhang, K. M.; Liu, W.; Tang, X. L.; Dou, W.; Ju, Z. H.; Li, Z. Q.; Liu, W. S. J. Mater. Chem. B 2015, 3, 3459.

[25] Song, K. C.; Kim, J. S.; Park, S. M.; Chuang, K.-C.; Ahn, S.; Chang, S.-K. Org. Lett. 2006, 8, 3413.

[26] Wang, K. N.; Feng W. J.; Wang, Y. B.; Cao, D. X.; Guan, R. F.; Yu, X. Y.; Wu, Q. Q. Inorg. Chem. Commun. 2016, 71, 102.

[27] Ghosh, K.; Kar, D. J. Inclusion Phenom. Macrocyclic Chem. 2013, $77,67$.

[28] Erdemir, S.; Tavakci, B.; Tabakci, M. Sens. Actuators, B 2016, 228, 109.

[29] Tang, L. J.; He, P.; Zhong, K. L.; Hou, S. H.; Bian, Y. J. Spectrochim. Acta, Part A 2016, 169, 246.

[30] Fan, C. L.; Luo, S. X.; Liu, R. RSC Adv. 2015, 5, 65321.

[31] Wen, H.; Huang, Q.; Yang, X.-F.; Li, H. Chem. Commun. 2013, 49, 4956.

[32] Santana, D. M.; Garcia-Bueno, R.; Garcia, G.; Piernas, M. J.; Perez, J.; Garcia, L.; Lopez-Garcia, I. Polyhedron 2013, 61, 161.

[33] Xu, Z. C.; Xiao, Y.; Qian, X. H.; Cui, J. N.; Cui, D. W. Org. Lett. 2005, 7, 889 .

[34] Wagh, Y. B.; Kuwar, A.; Sahoo, S. K.; Gallucci, J.; Dalal, D. S. RSC Adv. 2015, 5, 45528 .

[35] Qi, J. J.; Han, M. S.; Tung, C.-H. Bioorg. Med. Chem. Lett. 2012, 22, 1747.

[36] Arockiam, J. B.; Ayyanar, S. Sens. Actuators, B 2017, 242, 535

[37] Erdemir, S.; Ozcan, K. Talanta 2016, 158, 63.

[38] Liu, S.-D.; Zhang, L.-W.; Liu, X. New J. Chem. 2013, 37, 821.

[39] Das, S.; Aich, K.; Goswami, S.; Quah, C. K.; Fun, H.-K. New J. Chem. 2016, 40, 6414

[40] Nas, A.; Dilber, G.; Durmus, M.; Kantekin, H. Spectrochim. Acta, Part A 2015, 135, 55.

[41] Gogoi, A.; Samanta, S.; Das, G. Sens. Actuators, B 2014, 202, 788.

[42] Gogoi, A.; Das, G. RSC Adv. 2014, 4, 55689.

[43] Gogoi, A.; Mukherjee, S.; Ramesh, A.; Das, G. RSC Adv. 2015, 5, 63634.

[44] Jin, Y. Z.; Wang, S.; Zhang, Y. J.; Song, B. Sens. Actuators, B 2016, $225,167$.

[45] Kumar, M.; Kumar, N.; Bhalla, V. RSC Adv. 2013, 3, 1097.

[46] Yang, X. F.; Zhang, Y.; Li, Y. X.; Liu, X. L.; Mao, J. X.; Yuan, Y.; Cui, Y.; Sun, G. X.; Zhang, G. Y. RSC Adv. 2016, 6, 52004.

[47] Zhang, X. Y.; Chen, S. S.; Jin, S. H.; Lu, X. X.; Li, L. J.; Chen, X.; Shu, Q. H. Sens. Actuators, B 2016, 237, 367.

[48] Patil, R.; Moirangthem, A.; Butcher, R.; Singh, N.; Basu, A.; Tayade, K.; Fegade, U.; Hundiwale, D.; Kuwar, A. Dalton Trans. 2014, 43, 2895.

[49] Dhaka, G.; Kaur, N.; Singh, J. Supramol. Chem. 2015, 27, 654.

[50] Zhang, H. M.; Wu, Y. C.; You, J. Y.; Cao, L.; Ding, S.; Jiang, K.; Wang, Z. Y. Chin. J. Org. Chem. 2016, 36, 2559 (in Chinese). (张惠敏, 吴彦城, 尤佳宜, 曹梁, 丁沙, 蒋凯, 汪朝阳, 有机化 学, 2016, 36, 2559.)

[51] Liu, S. D.; Zhang, L. W.; Zhou, P. P.; Zan, W. Y.; Yao, X. J.; Yang, J. J.; Yang, Y. RSC Adv. 2015, 5, 19983.
[52] Zhang, X.; Liu, J. Y. Dyes Pigm. 2016, 125, 80.

[53] Shi, H. P.; Yang, J. W.; Dong, X. Q.; Fang, L.; Dong, C.; Choi, M. M F. Synth. Met. 2013, 179, 42.

[54] Dhaka, G.; Singh, J.; Kaur, N. Inorg. Chim. Acta 2016, 450, 380

[55] Erdemir, S.; Kocyigit, O. Sens. Actuators, B 2015, 221, 900.

[56] Wu, Y. C.; Huo, J. P.; Cao, L.; Ding, S.; Wang, L. Y.; Cao, D. R.; Wang, Z. Y. Sens. Actuators, B 2016, 237, 865.

[57] Wu, Y. C.; You, J. Y.; Jiang, K.; Xie, J. C.; Li, S. L.; Cao, D. R.; Wang, Z. Y. Dyes Pigm. 2017, 140, 47.

[58] Razi, S. S.; Gupta, R. C.; Ali, R.; Dwivedi, S. K.; Srivastava, P.; Misra, A. Sens. Actuators, B 2016, 236, 520.

[59] Hu, W.; Zeng, L. Y.; Wang, Y. Y.; Liu, Z. H.; Ye, X. X.; Li, C. Y. Analyst 2016, 141, 5450 .

[60] Goswami, S.; Das, A. K.; Manna, A.; Maity, A. K.; Fun, H. K.; Quah, C. K.; Saha, P. Tetrahedron Lett. 2014, 55, 2633.

[61] Vidya, B.; Iniya, M.; Sivaraman, G.; Sumesh, R. V.; Cheliappa, D. Sens. Actuators, B 2017, 242, 434.

[62] Goswami, S.; Manna, A.; Paul, S.; Aich, K.; Das, A. K.; Chakraborty, S. Tetrahedron Lett. 2013, 54, 1785.

[63] Tang, L. J.; Zou, Y. H.; Zhong, K. L.; Bian, Y. J. RSC Adv. 2016, 6 , 48351.

[64] Tang, Z.; Ding, X.-L.; Liu, Y.; Zhao, Z.-M.; Zhao, B.-X. RSC Adv. 2015, 5, 99664.

[65] Chen, S.; Hou, P.; Wang, J. X.; Song, X. Z. RSC Adv. 2012, 2, 10869

[66] Wang, J. L.; Long, L. P.; Xie, D. Chin. J. Appl. Chem. 2016, 33, 841 (in Chinese) (王姣亮，龙立平，谢丹，应用化学, 2016, 33, 841.)

[67] Zhang, Y. J.; Guan, L. M.; Yu, H.; Yan, Y. H.; Du, L. B.; Liu, Y.; Sun, M. T.; Huang, D. J.; Wang, S. H. Anal. Chem. 2016, 88, 4426.

[68] Geng, L. H.; Yang, X. F.; Zhong, Y. G.; Li, Z.; Li, H. Dyes Pigm. 2015, 120, 213.

[69] Liu, X. J.; Yang, Q. W.; Chen, W. Q.; Mo, L. N.; Chen, S.; Kang, J.; Song, X. Z. Org. Biomol. Chem. 2015, 13, 8663.

[70] Zhang, H. T.; Xie, Y. S.; Wang, P.; Chen, G. C.; Liu, R. C.; Lam, Y. W.; Hu, Y.; Zhu, Q.; Sun, H. Y. Talanta 2015, 135, 149.

[71] Fan, F. L.; Jing, J. Q.; Chen, X. M. Chin. J. Org. Chem. 2014, 34 2178 (in Chinese). (范方禄，靖金球，陈雪梅，有机化学, 2014, 34, 2178.)

[72] Das, S. K.; Lim, C. S.; Yang, S. Y.; Han, J. H.; Cho, B. R. Chem Commun. 2012, 48, 8395.

[73] Zhang, J. Y.; Guo, W. Chem. Commun. 2014, 50, 4214.

[74] Jiang, Y.; Wu, Q.; Chang, X.-J. Talanta 2014, 121, 122

[75] He, P.; Tang, L. J.; Zhong, K. L.; Hou, S. H.; Yan, X. M. Chin. J. Org. Chem. 2017, 37, 423 (in Chinese). (何平, 汤立军, 钟克利, 侯淑华, 燕小梅, 有机化学, 2017, 37, 423.)

[76] Garcia-Beltran, O.; Santos, J. G.; Fuentealba, S.; De la Torre, P.; Pavez, P.; Mena, N.; Nunez, M. T.; Aliaga, M. E. Tetrahedron Lett. 2015, 56, 2437.

[77] Paul, S.; Goswami, S.; Das Mukhopadhyay, C. New J. Chem. 2015, 39,8940 .

[78] Huang, Q.; Yang, X.-F.; Li, H. Dyes Pigm. 2013, 99, 871.

[79] Liu, Y.; Ding, Y.; Huang, J.; Zhang, X. F.; Fang, T. Y.; Zhang, Y. Y.; Zheng, X.; Yang, X. Dyes Pigm. 2017, 138, 112.

[80] Khandare, D. G.; Banerjee, M.; Gupta, R.; Kumar, N.; Ganguly, A.; Singh, D.; Chatterjee, A. RSC Adv. 2016, 6, 52790.

[81] Sun, W.; Li, W.-H.; Li, J.; Zhang, J.; Du, L.-P.; Li, M. Y. Tetrahedron Lett. 2012, 53, 2332.

[82] Kand, D.; Mandal, P. S.; Datar, A.; Talukdar, P. Dyes Pigm. 2014, 106,25

[83] Chen, X. Y.; Guo, L.; Zheng, C. G.; Gao, H. Y.; An, W. Chin. J. Appl. Chem. 2012, 29, 892 (in Chinese). (陈秀英，郭琳，郑昌戈，高海燕，安文，应用化学，2012，29, 892.)

[84] Chen, S.; Li, H. M.; Hou, P. Tetrahedron 2017, 73, 589.

[85] Rothweiler, U.; Stensen, W.; Brandsdal, B. O.; Isaksson, J.; Leeson, F. A.; Engh, R. A.; Svendsen, J. S. M. J. Med. Chem. 2016, 59 
9814.

[86] Kovalska, V. B.; Losytskyy, M. Y.; Tolmachev, O. I.; Slominskii, Y. L.; Segers-Nolten, G. M. J.; Subramaniam, V.; Yarmoluk, S. M. J. Fluoresc. 2012, 22, 1441.

[87] Ren, X. T.; Wang, F.; Lv, J.; Wei, T. W.; Zhang, W.; Wang, Y.; Chen, X. Q. Dyes Pigm. 2016, 129, 156.

[88] Zhang, D.; Chen, W.; Kang, J.-M.; Ye, Y.; Zhao, Y.-F.; Xian, M. Org. Biomol. Chem. 2014, 12, 6837.

[89] Liu, Z. M.; Feng, L.; Ge, G. B.; Lv, X.; Hou, J.; Cao, Y. F.; Cui, J. N.; Yang, L. Biosens. Bioelectron. 2014, 57, 30.

[90] Kim, T.; Kim, H.; Choi, Y.; Kim, Y. Chem. Commun. 2011, 47, 9825.

[91] Yang, X. F.; Guo, Y. X.; Strongin, R. M. Angew. Chem., Int. Ed. 2011, 50, 10690.

[92] Kim, Y.; Choi, M.; Seo, S.; Manjare, S. T.; Jon, S.; Churchill, D. G. RSC Adv. 2014, 4, 64183.

[93] Yang, X. F.; Huang, Q.; Zhong, Y. G.; Li, Z.; Li, H.; Lowry, M.; Escobedo, J. O.; Strongin, R. M. Chem. Sci. 2014, 5, 2177.

[94] Goswami, S.; Das, S.; Aich, K.; Pakhira, B.; Panja, S.; Mukherjee, S. K.; Sarkart, S. Org. Lett. 2013, 15, 5412.

[95] Patil, V. S.; Padalkar, V. S.; Tathe, A. B.; Sekar, N. Dyes Pigm. 2013, $98,507$.

[96] Liu, Z. P.; Zhang, C. L.; He, W. J.; Qian, F.; Yang, X. L.; Gao, X.; Guo, Z. J. New J. Chem. 2010, 34, 656.

[97] Zhan, Y.; Zhao, J. Y.; Yang, P.; Ye, W. J. RSC Adv. 2016, 6, 92144.

[98] Chao, J. B.; Liu, Y. H.; Sun, J. Y.; Fan, L.; Zhang, Y. B.; Tong, H. B.; Li, Z. Q. Sens. Actuators, B 2015, 221, 427.

[99] Liu, X. D.; Xu, Y.; Sun, R.; Xu, Y. J.; Lu, J. M.; Ge, J. F. Analyst 2013, 138,6542 .

[100] Sun, M. T.; Du, L. B.; Yu, H.; Zhang, K.; Liu, Y.; Wang, S. H. Talanta 2017, 162, 180.
[101] Muthusubramanian, S.; Saha, S. K. J. Lumin. 2012, 132, 2166.

[102] Sowmiya, M.; Purkayastha, P.; Tiwari, A. K.; Jaffer, S. S.; Saha, S. K. J. Photochem. Photobiol., A 2009, 205, 186.

[103] Tokar, V. P.; Losytskyy, M. Y.; Ohulchanskyy, T. Y.; Kryvorotenko, D. V.; Kovalska, V. B.; Balanda, A. O.; Dmytruk, I. M.; Prokopets, V. M.; Yarmoluk, S. M. J. Fluoresc. 2010, $20,865$.

[104] Sowmiya, M.; Tiwari, A. K.; Saha, S. K. J. Colloid Interface Sci. 2010, 344, 97.

[105] Gao. M.; Wang, L. C.; Chen, J. J.; Li, S. W.; Lu, G. H.; Wang, L.; Wang, Y. J.; Ren, L.; Qin, A. J.; Tang, B. Z. Chem. Eur. J. 2016, 22, 5107.

[106] Murale, D. P.; Kim, H.; Choi, W. S.; Churchill, D. G. Org. Lett. 2013, 15, 3946.

[107] Liu, C. Y.; Cao, Z. M.; Wang, Z. H.; Jia, P.; Liu, J.; Wang, Z. K.; Han, B. J.; Huang, X.; Li, X.; Zhu, B C. Sens. Actuators, B 2015 , $220,727$.

[108] Wu, Y. C.; You, J. Y.; Guan, L. T.; Shi, J.; Cao, L.; Wang, Z. Y. Chin. J. Org. Chem. 2015, 35, 2465 (in Chinese).

(吴彦城, 尤嘉宜, 关丽涛, 石杰, 曹梁, 汪朝阳, 有机化学, 2015, 35, 2465.)

[109] Mora, A. K.; Murudkar, S.; Alamelu, A.; Singh, P. K.; Chattopadhyay, S.; Nath, S. Chem. Eur. J. 2016, 22, 16505.

[110] Rajasekhar, K.; Narayanaswamy, N.; Murugan, N. A.; Kuang, G. L.; Argen, H.; Govindaraju, T. Sci. Rep. 2016, 6, 23668.

[111] Liu, Q. S.; Zhang, C. L.; Wang, X. Q.; Gong, S. W.; He, W. J.; Liu, Z. P. Chem. Asian J. 2016, 11, 202.

[112] Cao, L.; Xiong, J. F.; Wu, Y. C.; Li, M. B.; Xie, F.; Ma, Z. H.; Wang, Z. Y. Chin. J. Org. Chem. 2016, 36, 2053 (in Chinese). (曹梁, 吴彦城, 丁沙, 李铭冰, 谢芬, 马志晗, 汪朝阳, 有机化 学, 2016, 36, 2053.) 